\title{
Anisotropy of the de conductivity due to orbital-selective spin fluctuations in the nematic phase of iron superconductors
}

\author{
Raquel Fernández-Martín, ${ }^{1}$ Laura Fanfarillo,,$^{2, *}$ Lara Benfatto, ${ }^{3}$ and Belén Valenzuela ${ }^{1, \dagger}$ \\ ${ }^{1}$ Materials Science Factory, Instituto de Ciencia de Materiales de Madrid, ICMM-CSIC, Cantoblanco, E-28049 Madrid, Spain \\ ${ }^{2}$ CNR-IOM and International School for Advanced Studies (SISSA), Via Bonomea 265, I-34136, Trieste, Italy \\ ${ }^{3}$ ISC-CNR and Dep. of Physics, “Sapienza” University of Rome, P.le A. Moro 5, 00185, Rome, Italy
}

(Received 23 June 2018; revised manuscript received 8 February 2019; published 9 April 2019)

\begin{abstract}
We study the dc conductivity of iron-based superconductors within the orbital-selective spin fluctuation scenario. Within this approach, the anisotropy of spin fluctuations below the spin-nematic transition at $T_{S}$ is also responsible for the orbital ordering, induced by nematic self-energy corrections to the quasiparticle dispersion. As a consequence, the anisotropy of the dc conductivity below $T_{S}$ is determined not only by the anisotropy of the scattering rates as expected within a spin-nematic scenario, but also by the modification of the Fermi velocity due to the orbital reconstruction. More interestingly, it turns out that these two effects contribute to the dc-conductivity anisotropy with opposite signs. By using realistic band-structure parameters we compute the conductivity anisotropy for both 122 and FeSe compounds, discussing the possible origin of the different dc-conductivity anisotropy observed experimentally in these two families of iron-based superconductors.
\end{abstract}

DOI: 10.1103/PhysRevB.99.155117

\section{INTRODUCTION}

The driving force of electronic nematicity is one of the most intriguing puzzles in iron-based superconductors (IBS). The structural transition from tetragonal to orthorhombic at $T_{S}$ comprised the nematic phase characterized by a marked electronic anisotropy, much larger than the one expected by the structural transition itself [1]. In most iron pnictides the structural transition precedes or coincides with the magnetic transition at $T_{N}$. The proximity of the magnetic phase led to the proposal of the band spin-nematic scenario $[2,3]$ where the spin fluctuations (SF) at $\mathbf{Q}_{X}=(\pi, 0)$ and at $\mathbf{Q}_{Y}=(0, \pi)$ become anisotropic below $T_{S}$. The lack of long-range magnetic order in FeSe has cast some doubts on the validity of the spinnematic scenario in this compound. FeSe presents a nematic phase below the structural transition at $T_{S}=90 \mathrm{~K}$ down to the critical superconducting temperature $T_{c} \sim 9 \mathrm{~K}$. Even though a magnetic phase is not stabilized in temperature, sizable SF have been detected also in FeSe [4-8]. ARPES experiments in the nematic phase report a momentum-modulated orbital splitting between the $\Gamma$ and $M$ point of the Brillouin zone [9] that has been interpreted via both the orbital-ordering scenario [10-15] and the spin-nematic scenario [16]. In this situation two related questions arise: what is the role of the spin-orbital interplay and whether the origin of nematicity is universal in IBS or material dependent [17-19].

Resistivity anisotropy is a hallmark of nematicity in IBS. In detwinned electron (e)-doped 122 compounds $\Delta \rho=\rho_{x}-\rho_{y}<0$ is found below the structural transition [20-24] while detwinned hole (h)-doped compounds present the opposite anisotropy [25]. There is an on-going debate in

\footnotetext{
*laura.fanfarillo@sissa.it

†belenv@icmm.csic.es
}

the literature on whether the observed dc anisotropy is due to the anisotropy in the scattering rate or to the anisotropy in Fermi surface (FS) parameters [26-34]. In principle, within an orbital-ordering scenario the different occupation of the various orbitals affects mainly the FS [35-37], while within a spin-driven scenario the largest effect is expected to come from an anisotropy in the inelastic scattering rate [26,38-41]. Specifically, in the band spin-nematic scenario, depending on the FS shape and size, the band nesting is active at the so called hot spots on the FS, where the scattering rate is maximum. It has been argued that the location of the hot spots could explain the different signs between e-doped compounds and h-doped compounds [25] in pnictides. Besides the spin-nematic or orbital order scenario, further attempts to explain the dc anisotropy in pnictides taking into account the spin-orbital interplay have been performed using the effective spin-fermion model [42] or the multiorbital microscopic model in the magnetic phase [43-45].

Recent experiments in $\mathrm{FeSe}$ have found the opposite anisotropy with respect to the e-doped 122 compounds [46], i.e., $\Delta \rho=\rho_{x}-\rho_{y}>0$. Given the significant FS reconstruction observed in the nematic phase of FeSe [9], we need to revise the role of the scattering rate and velocity anisotropies taking into account the spin-orbital interplay in order to theoretically address both pnictides and FeSe. Within an orbitalordering scenario, the opposite anisotropy of the resistivity of 122 and FeSe compounds in the nematic phase has been ascribed to the orbital-dependent inelastic quasiparticle scattering by orbital-dependent SF [47]. However, an analogous study of the dc conductivity anisotropy within a spin-nematic scenario accounting for the spin-orbital interplay and able to address pnictides and FeSe, is still missing.

The aim of this work is then to provide an interpretation for the observed differences, using as a starting point the orbitalselective spin fluctuation (OSSF) model. The model, derived 


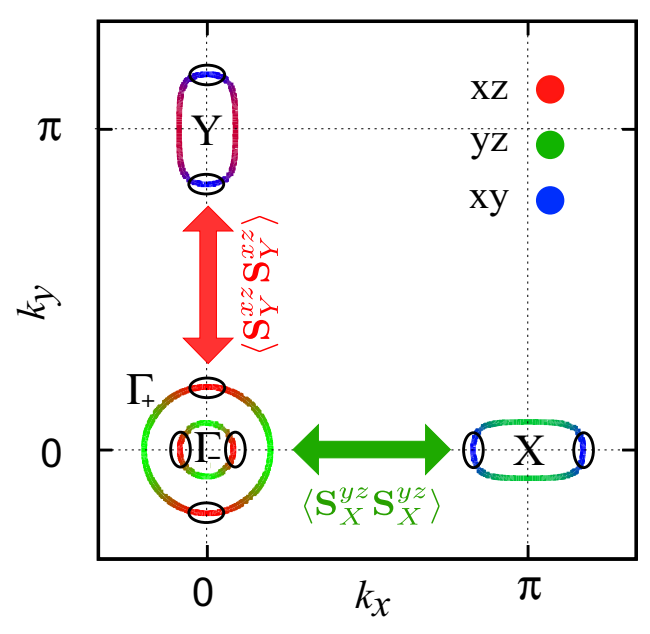

FIG. 1. General sketch of the orbital content of the Fermi surface of the four-pocket model for iron superconductors. The green and red arrows show the OSSF with $y z$ content in the $x$ direction and $x z$ content in the $y$ direction. Cold spots, where the scattering rate is minimum, are shown by a circle and they are found on the $x y$ and $x z$ orbitals in the nematic phase due to anisotropic self-energy corrections. See the text.

in the itinerant approach [19], exploits the original idea of the orbital-selective character of the SF in IBS discussed in Ref. [48]. Within the OSSF model, due to the orbital composition of the FS, the SF peaked at $\mathbf{Q}_{X}=(\pi, 0)$ involve only the $y z$ orbital, while the $\mathrm{SF}$ at $\mathbf{Q}_{Y}=(0, \pi)$ involve only the $x z$ orbital, Fig. 1 . As in the spin-nematic scenario, the nematic phase emerges when SF at $\mathbf{Q}_{X}=(\pi, 0)$ and at $\mathbf{Q}_{Y}=(0, \pi)$ become anisotropic, however in the OSSF model such anisotropy directly affects the $y z / x z$ orbital symmetry. The OSSF is a minimal model that explains successfully the enhanced nematic tendency of FeSe as compared to 122 systems [19] and clarifies controversial experimental issues in FeSe such as the temperature evolution of the FS of FeSe and the odd orbital ordering observed by ARPES experiments [16], the decrease of the nematic critical temperature, and the emergence of magnetism in FeSe with pressure [19] as recently observed in $[9,49,50]$. Moreover, the analysis of the superconductivity mediated by anisotropic OSSF [51] successfully account for the enigmatic anisotropy of the superconducting gap revealed by STM [52] and ARPES [53-55] experiments in FeSe.

In this work we analyze, within the OSSF model, the effect of anisotropic self-energy corrections on the conductivity anisotropy in the nematic phase of IBS. In contrast to the band spin-nematic scenario [2,3], where just the scattering rate contributes to the conductivity anisotropy, we found that also the velocity contributes. The contribution of the scattering rate to the resistivity anisotropy is dominated by the location of the cold spots where the scattering rate is minimum (see Fig. 1), which, within our model, is determined by the orbital composition of the FS and by the spin-orbital interplay of the OSSF. The contribution of the velocity to the resistivity anisotropy is counterintuitive and opposite to the one of the scattering rate. We find indeed that the conductivity is larger in the direction where the self-energy is also larger. This interesting new effect is due to an orbital character exchange in the pockets arising from the OSSF self-energy in the nematic phase. Our study shows that the sign of the anisotropy of the dc conductivity depends on whether scattering rate or velocity anisotropy dominates on each pocket, as well as other parameters such as the ellipticity and the quasiparticle renormalization due to local interactions [56]. Thus, different experimental results among the various families of IBS can be explained within the same OSSF scenario.

The structure of the paper is the following. In Sec. II we introduce the OSSF model. In Sec. III we outline the calculation of the dc conductivity. In Sec. III A we derive analytical expressions for the dc anisotropy within the perfectly nested parabolic-band approximation. In Sec. III B we discuss numerical results obtained using realistic parameters for 122 compounds and $\mathrm{FeSe}$, i.e., accounting for the effects of spin-orbit coupling and elliptical e pockets. In Sec. III C we discuss our results in connection to experiments in IBS. In Sec. IV we summarize our results and draw the conclusions of our work.

\section{MODEL}

The OSSF low-energy model has been derived in detail in Ref. [19]. Here we summarize the main features of the model, further details can be found in Appendix A. The starting point is a general four-pocket model with two $\mathrm{h}$ pockets at $\Gamma$, denoted as $\Gamma^{ \pm}$, and two e pockets at $X$ and $Y$. The model can be easily adapted to describe different compounds among the 122 and 11 families. The kinetic part of the Hamiltonian is derived following the low-energy approach considered in Ref. [57], where each pocket is described using a spinor representation in the orbital space:

$$
H_{0}^{l}=\sum_{\mathbf{k}, \sigma} \psi_{\mathbf{k} \sigma}^{\dagger l} \hat{H}_{0 \mathbf{k}}^{l} \psi_{\mathbf{k} \sigma}^{l}, \quad \hat{H}_{0 \mathbf{k}}^{l}=h_{0 \mathbf{k}}^{l} \hat{\tau_{0}}+\vec{h}_{\mathbf{k}}^{l} \cdot \overrightarrow{\hat{\tau}}
$$

where $l=\Gamma, X, Y$ and $\hat{\tau}$ are the Pauli matrices representing the orbital pseudospin. The spinors are defined as $\psi_{\mathbf{k} \sigma}^{\Gamma}=$ $\left(c_{\mathbf{k}, \sigma}^{y z}, c_{\mathbf{k} \sigma}^{x z}\right)$ and $\psi_{\mathbf{k} \sigma}^{X / Y}=\left(c_{\mathbf{k} \sigma}^{y z / x z}, c_{\mathbf{k} \sigma}^{x y}\right)$. Rotating the Hamiltonian into the band basis we have

$$
H_{0}^{l}=\sum_{\mathbf{k}, \sigma} E_{\mathbf{k}}^{l \pm} c_{\mathbf{k} \sigma}^{\dagger l \pm} c_{\mathbf{k} \sigma}^{l \pm}
$$

where $E_{\mathbf{k}}^{l \pm}=h_{0 \mathbf{k}}^{l} \pm h_{\mathbf{k}}^{l}, h_{\mathbf{k}}^{l}=\left|\vec{h}_{\mathbf{k}}^{l}\right|$ are the band dispersions. The fermionic band operators $c^{l \pm}$ are obtained rotating the orbital spinors via an unitary matrix $\hat{\mathcal{U}}^{l}$. Explicitly for the $\mathrm{h}$ pockets at $\Gamma, c^{\Gamma \pm} \equiv h^{ \pm}$we have

$$
\left(\begin{array}{l}
h^{+} \\
h^{-}
\end{array}\right)=\left(\begin{array}{cc}
u^{\Gamma} & -v^{\Gamma} \\
v^{* \Gamma} & u^{* \Gamma}
\end{array}\right)\left(\begin{array}{l}
c^{y z} \\
c^{x z}
\end{array}\right)=\hat{\mathcal{U}}^{\Gamma}\left(\begin{array}{l}
c^{y z} \\
c^{x z}
\end{array}\right)
$$

where we have dropped the momentum and spin indices for simplicity. Analogous expressions hold for the $X / Y$ e-pockets fermionic operators $c^{X / Y \pm} \equiv e^{X / Y \pm}$. Since only the $E^{X / Y+}$ band crosses the Fermi level at $X / Y$, in the following we will drop the + subscript from $e^{X / Y}$.

The interacting Hamiltonian simplifies substantially once the spin-exchange interaction is projected at low-energy $[19,48]$. The generic intraorbital spin operator reads 
$\mathbf{S}_{\mathbf{q}}^{\eta}=\sum_{\mathbf{k} \alpha \beta}\left(c_{\mathbf{k} \alpha}^{\eta \dagger} \vec{\sigma}_{\alpha \beta} c_{\mathbf{k}+\mathbf{q} \beta}^{\eta}\right)$, with $\eta$ the orbital index and $\vec{\sigma}_{\alpha \beta}$ the Pauli matrices for the spin. Thus, taking into account the orbital composition of the FS shown in Fig. 1, the relevant intraorbital spin operator occurs at momenta $\mathbf{q}$ near $\mathbf{Q}_{X}=$ $(\pi, 0)$ and $\mathbf{Q}_{Y}=(0, \pi)$, which connect a $\mathrm{h}$ pocket with the $X$ and $Y$ e pockets, respectively. Since the only common orbital is the $y z / x z$ along the $x / y$ direction the spin interaction reduces to

$$
H_{\mathrm{int}}=-\frac{\tilde{U}}{2} \sum_{\mathbf{q}^{\prime}} \mathbf{S}_{X / Y}^{y z / x z} \cdot \mathbf{S}_{X / Y}^{y z / x z}
$$

Here $\tilde{U}$ is the intraorbital interaction renormalized at low energy and $\mathbf{S}_{X / Y}^{\eta} \equiv \vec{S}_{\mathbf{q}=\mathbf{Q}_{X / Y}}^{\eta}$. The relevant magnetic fluctuations peaked at $\mathbf{Q}_{X} / \mathbf{Q}_{Y}$ are orbital selective, as sketched in Fig. 1, having only $y z / x z$ orbital character:

$$
\begin{array}{r}
\langle\mathbf{S} \cdot \mathbf{S}\rangle\left(\mathbf{Q}_{X}\right) \Rightarrow\left\langle\mathbf{S}_{X}^{y z} \cdot \mathbf{S}_{X}^{y z}\right\rangle, \\
\langle\mathbf{S} \cdot \mathbf{S}\rangle\left(\mathbf{Q}_{Y}\right) \Rightarrow\left\langle\mathbf{S}_{Y}^{x z} \cdot \mathbf{S}_{Y}^{x z}\right\rangle .
\end{array}
$$

The SF exchange between h- and e-like pockets renormalizes the quasiparticles via single-particle self-energy corrections. Within the OSSF model, due to the orbital-selective nature of SF, this mechanism is also orbital dependent. The self-energy corrections in the orbital basis can be computed within an Eliashberg-like treatment [16] for each pocket. For the h pockets at $\Gamma$ we find

$$
\hat{\Sigma}^{\Gamma}(\omega)=\left(\begin{array}{cc}
\Sigma_{y z}^{\Gamma}(\omega) & 0 \\
0 & \Sigma_{x z}^{\Gamma}(\omega)
\end{array}\right)=\Sigma_{0}^{\Gamma}(\omega) \hat{\tau}_{0}+\Sigma_{3}^{\Gamma}(\omega) \hat{\tau}_{3},
$$

with $\quad \Sigma_{0}^{\Gamma}(\omega)=\left[\Sigma_{y z}^{\Gamma}(\omega)+\Sigma_{x z}^{\Gamma}(\omega)\right] / 2, \quad \Sigma_{3}^{\Gamma}(\omega)=\left[\Sigma_{y z}^{\Gamma}(\omega)-\right.$ $\left.\Sigma_{x z}^{\Gamma}(\omega)\right] / 2$, while for the e pockets we have

$$
\hat{\Sigma}^{X / Y}(\omega)=\left(\begin{array}{cc}
\Sigma_{y z / x z}^{X / Y}(\omega) & 0 \\
0 & 0
\end{array}\right)=\Sigma_{0}^{X / Y}(\omega) \hat{\tau}_{0}+\Sigma_{3}^{X / Y}(\omega) \hat{\tau_{3}},
$$

with $\Sigma_{0}^{X / Y}(\omega)=\Sigma_{3}^{X / Y}(\omega)=\Sigma_{y z / x z}^{X / Y}(\omega) / 2$. In the tetragonal state, above the structural transition, the isotropic SF lead to equivalent self-energies for the $C_{4}$ symmetric $x z / y z$ orbitals, i.e., $\Sigma_{3}^{\Gamma}=0$ and $\Sigma_{3}^{X}=\Sigma_{3}^{Y}$. In the nematic phase the anisotropy of the OSSF below $T_{S}$ generates a differentiation of the $x z / y z$ self-energy corrections

$$
\begin{aligned}
& \Sigma_{3}^{\Gamma}(\omega) \neq 0, \\
& \Sigma_{3}^{X}(\omega) \neq \Sigma_{3}^{Y}(\omega),
\end{aligned}
$$

and gives rise to an effective orbital nematicity. Equations (7) and (8) are generic but their specific values are material dependent and for FeSe were calculated at the RPA level in Ref. [16]. The dressed Green's functions are obtained via the Dyson equation $\hat{G}^{l^{-1}}(\mathbf{k}, \omega)=\hat{G}_{0}^{l^{-1}}(\mathbf{k}, \omega)-\hat{\Sigma}^{l}(\omega)$, where $\hat{G}_{0}^{l^{-1}}(\mathbf{k}, \omega)=\omega \hat{\mathbb{1}}-\hat{H}_{0}^{l}(\mathbf{k})$. We diagonalize the renormalized Green's function via the unitary transformation $\hat{\mathcal{U}}_{R}^{l}(\mathbf{k}, \omega)$ defined as

$$
\hat{G}^{l}(\mathbf{k}, \omega)=\hat{\mathcal{U}}_{R}^{l}(\mathbf{k}, \omega)\left[\omega \hat{\mathbb{1}}-\hat{\Lambda}_{R}^{l}(\mathbf{k}, \omega)\right]^{-1} \hat{\mathcal{U}}_{R}^{l^{-1}}(\mathbf{k}, \omega),
$$

where $\hat{\Lambda}_{R}^{l}=\operatorname{diag}\left(E_{R}^{l+}, E_{R}^{l-}\right)$ and

$$
\begin{aligned}
E_{R}^{l_{ \pm}}(\mathbf{k}, \omega) & =h_{\mathbf{k} 0}^{l}+\Sigma_{0}^{l}(\omega) \pm h_{R \mathbf{k}, \omega}^{l}, \\
h_{R \mathbf{k}, \omega}^{l} & =\sqrt{\left[h_{3}^{l}+\Sigma_{3}^{l}(\omega)\right]^{2}+\left(h_{1}^{l}\right)^{2}+\left(h_{2}^{l}\right)^{2}} .
\end{aligned}
$$

Since the self-energy is a complex function, Eq. (11) accounts both for the renormalization of the band dispersion

$$
\epsilon_{R}^{l_{ \pm}}(\mathbf{k}, \omega)=\operatorname{Re} E_{R}^{l_{ \pm}}(\mathbf{k}, \omega)
$$

and for the renormalized scattering rate

$$
\Gamma_{R}^{l_{ \pm}}(\mathbf{k}, \omega)=\delta \Gamma+\left|\operatorname{Im} E_{R}^{l_{ \pm}}(\mathbf{k}, \omega)\right|,
$$

where we also added a residual constant broadening term $\delta \Gamma$. Equation (13) establishes a connection between the scattering rate and the self-energy renormalizations contained in $E_{R}^{l_{ \pm}}$. The qualitative behavior of the self-energies in the nematic phase allows us to easily localize the minimum value of $\Gamma_{R}^{l_{ \pm}}$on the FS, i.e., the cold spots shown in Fig. 1. As we discussed in Ref. [16], the reconstruction of the FS below $T_{S}$ is consistent with the Ising-nematic spin fluctuations being bigger at $\mathbf{Q}_{X}$ than at $\mathbf{Q}_{Y}$. This implies that self-energy corrections are stronger on the $y z$ orbital than on the $x z$ one. As a consequence on the $h$ pockets the smaller scattering rate corresponds to the $x z$ orbital. On the e pockets instead, the smaller scattering rate is found for the $x y$ orbital, given the absence of $x y$-SF within our model. The result is an example of the spin-orbital interplay retained by the OSSF approach that allows us to directly link the cold spots position with the FS orbital character and is not present in the band-based spin-nematic scenario [2].

The rotation matrix $\hat{\mathcal{U}}_{R}^{l}(\mathbf{k}, \omega)$ in Eq. (10) has a structure analogous to Eq. (3). The renormalized coherence factors $u_{R}^{l}$ and $v_{R}^{l}$, whose exact expressions are given in Appendix A, depend now on the self-energies Eqs. (7) and (8). The approximated expressions for $u_{R \mathbf{k}}^{l}(\omega), v_{R \mathbf{k}}^{l}(\omega)$ obtained at low energy $\omega$ by expanding in $\Sigma_{3}^{l}$ at the first order read

$$
\begin{aligned}
& \left|u_{R}^{l}(\omega)\right|^{2}=\left|u^{l}\right|^{2}\left(1+\frac{2 \operatorname{Re} \Sigma_{3}^{l}(\omega)}{h^{l}}\left|v^{l}\right|^{2}\right), \\
& \left|v_{R}^{l}(\omega)\right|^{2}=\left|v^{l}\right|^{2}\left(1-\frac{2 \operatorname{Re} \Sigma_{3}^{l}(\omega)}{h^{l}}\left|u^{l}\right|^{2}\right),
\end{aligned}
$$

where we neglected the imaginary part of the self-energy which approaches zero at low energy and low temperature. $u^{l}$ and $v^{l}$ are the bare coherence factors appearing in Eq. (3) and detailed in Appendix A. From Eq. (14) one sees that the correction term $\sim \operatorname{Re} \Sigma_{3}^{l}(\omega)$ mixes the orbital character in each pocket, i.e., contribute to $u_{R}^{l}$ with a term proportional to $v^{l}$ and vice versa. This effect of the OSSF self-energy in the coherence factors will have important consequences for the renormalized velocities as we will see in the following section.

\section{DC CONDUCTIVITY}

The dc conductivity is the $\Omega \rightarrow 0$ limit of the longitudinal optical conductivity given by

$$
\operatorname{Re} \sigma_{\alpha}(\Omega)=-\frac{e^{2}}{V} \frac{\operatorname{Im} \Pi_{\alpha}\left(\mathbf{q}=0, i \Omega_{m} \rightarrow \Omega\right)}{\Omega},
$$


where $\alpha=x, y, V$ is the unit-cell volume and $\Pi_{\alpha}$ is the current-current correlation function that in the bubble approximation reads

$$
\begin{aligned}
\Pi_{\alpha}\left(\mathbf{q}, i \Omega_{m}\right)= & 2 T \sum_{l \mathbf{k} n} \operatorname{Tr}\left[\hat{G}^{l}\left(\mathbf{k}-\mathbf{q} / 2, i \omega_{n}\right) \hat{V}_{\mathbf{k}_{\alpha}}^{l}\right. \\
& \left.\times \hat{G}^{l}\left(\mathbf{k}+\mathbf{q} / 2, i \omega_{n}+i \Omega_{m}\right) \hat{V}_{\mathbf{k}_{\alpha}}^{l}\right] .
\end{aligned}
$$

$\hat{V}_{\mathbf{k}_{\alpha}}^{l}=\partial_{\mathbf{k}_{\alpha}} \hat{H}_{0}^{l}$ is the bare velocity operator and $\hat{G}^{l}\left(\mathbf{k}, i \omega_{n}\right)$ is the renormalized Green's function defined in Eq. (10). We rotate Eq. (16) in the band basis using Eq. (10), perform the trace, and take the $\Omega \rightarrow 0$ limit. The $\alpha=x, y$ component of the dc conductivity in the band basis, $\sigma_{\mathrm{dc} \alpha}=\sum_{l_{ \pm}} \sigma_{\alpha}^{l_{ \pm}}$, is obtained from the sum over all the pockets $l_{ \pm}=\Gamma_{+}, \Gamma_{-}, X, Y$. The pocket conductivity reads (see Appendix B for further details)

$$
\sigma_{\alpha}^{l_{ \pm}}=\frac{2 \pi e^{2}}{N} \sum_{\mathbf{k}} \int_{-\infty}^{\infty} d \omega\left(-\frac{\partial f(\omega)}{\partial \omega}\right)\left[V_{R \mathbf{k}_{\alpha}}^{l_{ \pm}}(\omega)\right]^{2}\left[A_{\mathbf{k}}^{l_{ \pm}}(\omega)\right]^{2}
$$

In this basis the spectral function is diagonal

$$
A_{\mathbf{k}}^{l_{ \pm}}(\omega)=\frac{1}{\pi} \frac{\Gamma_{R}^{l_{ \pm}}(\omega)}{\left[\Gamma_{R}^{l_{ \pm}}(\omega)\right]^{2}+\left[\omega-\epsilon_{R \mathbf{k}}^{l_{ \pm}}(\omega)\right]^{2}}
$$

with $\Gamma_{R}^{l_{ \pm}}(\omega)$ and $\epsilon_{R \mathbf{k}}^{l_{ \pm}}(\omega)$ defined in Eqs. (12) and (13). $V_{R \mathbf{k} \alpha}^{l_{ \pm}}$ in Eq. (17) is the bare velocity operator rotated into the band basis. As a consequence of the orbital structure we have

$$
V_{R \mathbf{k} \alpha}^{l_{ \pm}}=V_{\mathbf{k} \alpha}^{l_{11}}\left|u_{R}^{l}\right|^{2} \pm V_{\mathbf{k} \alpha}^{l_{12}} u_{R}^{* l} v_{R}^{* l} \pm V_{\mathbf{k} \alpha}^{l_{21}} u_{R}^{l} v_{R}^{l}+V_{\mathbf{k} \alpha}^{l_{22}}\left|v_{R}^{l}\right|^{2} .
$$

Hereafter we omit the dependence on $\omega$ for simplicity. $V_{\mathbf{k} \alpha}^{l_{\eta \eta^{\prime}}}$ are the $\eta \eta^{\prime}$ component of the velocity and $(u / v)_{R}^{l}$ are the renormalized coherence factors. Via the coherence factors $V_{R \mathbf{k} \alpha}^{l_{ \pm}}$depends on the $\hat{\tau}_{3}$ component of the self-energies $\Sigma_{3}^{l}(\omega)$ that mixes the orbital content of each pocket. This effect can be easily understood considering the approximated expressions in Eq. (14). By neglecting the imaginary part of the self-energy in $(u / v)_{R}^{l}$, Eq. (19) can also be written as

$$
V_{R \mathbf{k} \alpha}^{l_{ \pm}}=\partial \epsilon_{R}^{l_{ \pm}}(\mathbf{k}) / \partial k_{\alpha} .
$$

In the $T \rightarrow 0$ limit we can approximate the Fermi function with a $\delta(\omega)$ which selects only states at the Fermi level $\omega=0$. By further assuming $\Gamma_{R}^{l_{ \pm}}$to be small we can also approximate the spectral functions with a delta function and Eq. (17) reduces to

$$
\sigma_{\alpha}^{l_{ \pm}}=\frac{e^{2}}{N} \sum_{\mathbf{k}} \frac{\left(V_{R \mathbf{k}_{\alpha}}^{l_{ \pm}}\right)^{2}}{\Gamma_{R \mathbf{k}}^{l_{ \pm}}} \delta\left(\epsilon_{R \mathbf{k}}^{l_{ \pm}}\right)
$$

\section{A. Analytical calculation}

To gain physical insight on the dc anisotropy and disentangle the effect of the velocity and scattering rate in Eq. (21), we estimate analytically $\sigma_{\alpha}$. We approximate the $\mathrm{h}$ and e bands with perfectly nested parabolic bands and assume that the nematic order is small enough to allow one for a perturbative expansion of the renormalized energy $E_{R}^{l \pm}$. We use a symmetric nematic splitting around the isotropic value $\Sigma_{0}^{l}$ in the tetragonal phase. By expanding Eq. (11) at first order in the $\tau_{3}$ self-energy component we can estimate analytically for each pocket $V_{R \mathbf{k}_{\alpha}}^{l_{ \pm}}$and $\Gamma_{R \mathbf{k}}^{l_{ \pm}}$via Eqs. (12), (13), and (20).

Deriving for example with respect to $k_{x}$, the renormalized energy of the pocket $\Gamma_{+}$, we find

$$
V_{R \mathbf{k} x}^{\Gamma_{+}}=-\frac{k \cos \theta}{m^{\Gamma_{+}}}+4 \operatorname{Re} \Sigma_{3}^{\Gamma_{+}} \sin ^{2} \theta \frac{k \cos \theta}{k^{2}},
$$

where $m^{\Gamma_{+}}$is the bare mass of the $\Gamma_{+}$pocket whose definition in terms of the Hamiltonian parameters is given in Appendix B. The first term on the right-hand side of Eq. (22), is the $x$ component of the bare velocity, while the second term $O\left(\operatorname{Re} \Sigma_{3}^{\Gamma}\right)$ is an additional contribution due to the orbital mixing induced by the nematic order as expected from the $(u, v)_{R}^{l}$ factors in Eq. (19). To compute the $\mathbf{k}$ integration in Eq. (21) we use the delta function and evaluate $V_{R \mathbf{k} x}^{\Gamma_{+}}$ at the renormalized FS. Notice that in the nematic phase $k_{F}^{\Gamma_{+}}(\theta)$ is no longer constant but gets deformed because of the anisotropic self-energy renormalization. This effect is also of order $O\left(\operatorname{Re} \Sigma_{3}^{\Gamma}\right)$ and has to be taken into account. We estimate the change in the Fermi wave vector at the first order in the self-energy. Replacing the expression of $k_{F}^{\Gamma_{+}}(\theta)$ into Eq. (22) we find

$$
V_{R x}^{\Gamma_{+}}=V_{0_{x}}^{\Gamma_{+}}\left(1+\cos 2 \theta \frac{\operatorname{Re} \Sigma_{3}^{\Gamma}}{2 \epsilon_{0}^{h}}-4 \sin ^{2} \theta \frac{\operatorname{Re} \Sigma_{3}^{\Gamma}}{2 \epsilon_{0}^{h}}\right),
$$

where $V_{0 x}^{\Gamma_{+}}=-k_{0} \Gamma_{F x}^{\Gamma_{+}} / m^{\Gamma_{+}}$and $\epsilon_{0}^{h}=\epsilon^{\Gamma}+\operatorname{Re} \Sigma_{0}^{\Gamma}$ are the velocity and the Fermi energy in the tetragonal phase, respectively. From Eq. (23) one sees that the bare Fermi velocity in the nematic phase has two contributions $O\left(\operatorname{Re} \Sigma_{3}^{\Gamma}\right)$ opposite in sign: the first one is due to the change in $k_{F}^{\Gamma_{+}}$, while the second one comes from the orbital mixing produced by the nematic order. Analogous calculation of the velocity contributions along $y$ for the $\Gamma_{+}$as well as for the other pockets lead to similar expressions [see Eq. (B12)] with the band velocity of the tetragonal phase renormalized by two additional contributions $O\left(\operatorname{Re} \Sigma_{3}^{l}\right)$ of opposite sign. The scattering rate is analytically estimated from Eq. (13) using again the expansion of $E_{R}^{\Gamma_{+}}$at the first order in $\Sigma_{3}^{l}$ :

$$
\Gamma_{R}^{\Gamma_{+}}(\theta)=\Gamma_{0}^{h}+\cos 2 \theta\left|\operatorname{Im} \Sigma_{3}^{\Gamma}\right| .
$$

Here we separate the tetragonal phase scattering rate $\Gamma_{0}^{h}=$ $\delta \Gamma+\left|\operatorname{Im} \Sigma_{0}^{\Gamma}\right|$ from the the angular-dependent correction due to the nematic effect $\sim \operatorname{Im} \Sigma_{3}^{\Gamma}$. The explicit expression of the $\Gamma_{+}$pocket dc conductivity follows from Eq. (21) using Eqs. (23) and (24):

$$
\begin{aligned}
\sigma_{x}^{\Gamma_{+}} & =\sigma^{h}\left(1+\frac{\operatorname{Re} \Sigma_{3}^{\Gamma}}{2 \epsilon_{0}^{h}}-\frac{\operatorname{Re} \Sigma_{3}^{\Gamma}}{\epsilon_{0}^{h}}-\frac{\left|\operatorname{Im} \Sigma_{3}^{\Gamma}\right|}{2 \Gamma_{0}^{h}}\right) \\
& =\sigma^{h}\left(1-\frac{\Phi^{h}}{2 \epsilon_{0}^{h}}+\frac{\Phi^{h}}{\epsilon_{0}^{h}}-\frac{\Delta \Gamma^{h}}{2 \Gamma_{0}^{h}}\right)
\end{aligned}
$$

$\epsilon_{0}^{h}, \Gamma_{0}^{h}$, and $\sigma^{h}=e^{2} \epsilon_{0}^{h} /(2 \pi \hbar) \Gamma_{0}^{h}$ are, respectively, the Fermi energy, the scattering rate, and the dc conductivity in the 
tetragonal phase. We also defined the real and imaginary part of the nematic order parameter for the $h$ pockets $\left(\Phi^{h}, \Delta \Gamma^{h}\right)$ as

$$
\begin{aligned}
\Phi^{h} & \equiv \frac{\operatorname{Re} \Sigma_{x z}^{\Gamma}-\operatorname{Re} \Sigma_{y z}^{\Gamma}}{2}=-\operatorname{Re} \Sigma_{3}^{\Gamma}, \\
\Delta \Gamma^{h} & \equiv\left|\operatorname{Im} \Sigma_{3}^{\Gamma}\right|,
\end{aligned}
$$

taking also into account that stronger spin fluctuation at $\mathbf{Q}_{X}$ implies $\operatorname{Re} \Sigma_{3}^{\Gamma}<0$, so that now the nematic order parameters are all positive defined.

Performing analogous calculations (see Appendix B) we derive the dc conductivities along $x$ and $y$ for each pocket. For the $\mathrm{h}$ pockets we find

$$
\begin{aligned}
& \sigma_{x / y}^{\Gamma_{+}}=\sigma^{h}\left(1 \mp \frac{\Phi^{h}}{2 \epsilon_{0}^{h}} \pm \frac{\Phi^{h}}{\epsilon_{0}^{h}} \mp \frac{\Delta \Gamma^{h}}{2 \Gamma_{0}^{h}}\right), \\
& \sigma_{x / y}^{\Gamma_{-}}=\sigma^{h}\left(1 \pm \frac{\Phi^{h}}{2 \epsilon_{0}^{h}} \mp \frac{\Phi^{h}}{\epsilon_{0}^{h}} \pm \frac{\Delta \Gamma^{h}}{2 \Gamma_{0}^{h}}\right) .
\end{aligned}
$$

In the absence of spin-orbit interaction the $\mathrm{h}$ pockets have the same $\epsilon_{0}^{h}$, so they also have the same conductivity $\sigma^{h}$ in the tetragonal phase. Additional terms proportional to $\Phi^{h}$ and $\Delta \Gamma^{h}$ arise in the nematic phase and make the conductivity different for the two $h$ pockets. As extensively discussed within the calculation of the velocity operator for the $\Gamma_{+}$ pocket in Eq. (23), the nematic order has two opposite effects $O\left(\Phi^{h}\right)$ in the velocity and this is reflected into the pocket dc conductivity anisotropy as one sees from Eq. (27). The first correction comes directly from the $k_{F}^{\Gamma_{ \pm}}$changes due to the nematic FS reconstruction, while the second one, opposite in sign, is due to the orbital mixing. Notice that this last term also determines the overall sign of the correction $\sim \Phi^{h}$ in each pocket. Due to the $x z / y z$ orbital arrangement of the $\Gamma_{ \pm} \mathrm{FS}$, the two h pockets contribute with opposite sign to the conductivity anisotropy, i.e., in Eq. (27) we find the same sign of the nematic terms in the conductivity along $x$ of the $\Gamma_{+}$pocket and in the conductivity along $y$ of the $\Gamma_{-}$one. In particular the opposite sign of the contribution $O\left(\Delta \Gamma^{h}\right)$ giving negative/positive anisotropy for the $\Gamma_{+/-}$pocket is a direct consequence of the cold-spots physics, Fig. 1, from where we can easily infer the sign of the anisotropic contribution for $\Gamma_{ \pm}$having in mind that lower scattering implies a bigger conductivity. By computing the h-dc conductivity anisotropy, $\Delta \sigma^{h_{ \pm}} \equiv \sigma_{x}^{\Gamma_{ \pm}}-\sigma_{y}^{\Gamma^{ \pm}}$, we find

$$
\begin{aligned}
& \Delta \sigma^{h+}=\sigma^{h}\left(\frac{\Phi^{h}}{\epsilon_{0}^{h}}-\frac{\Delta \Gamma^{h}}{\Gamma_{0}^{h}}\right), \\
& \Delta \sigma^{h-}=\sigma^{h}\left(-\frac{\Phi^{h}}{\epsilon_{0}^{h}}+\frac{\Delta \Gamma^{h}}{\Gamma_{0}^{h}}\right) .
\end{aligned}
$$

The dc conductivity components for the e pocket at $X$ read

$$
\begin{aligned}
& \sigma_{x}^{X}=\sigma^{e}\left(1-\frac{\operatorname{Re} \Sigma_{y x}^{X}}{4 \epsilon_{0}^{e}}+\frac{\left|\operatorname{Im} \Sigma_{y z}^{X}\right|}{4 \Gamma_{0}^{e}}\right), \\
& \sigma_{y}^{X}=\sigma^{e}\left(1+\frac{3 \operatorname{Re} \Sigma_{y x}^{X}}{4 \epsilon_{0}^{e}}-\frac{\left|\operatorname{Im} \Sigma_{y z}^{X}\right|}{4 \Gamma_{0}^{e}}\right) .
\end{aligned}
$$

As already done in Eq. (25) we defined the nematic correction with respect to the tetragonal $x / y$ dc conductivities. $\sigma_{x / y}^{e}$ are both equivalent to $\sigma^{e}=e^{2} \epsilon_{0}^{e} /(2 \pi \hbar) \Gamma_{0}^{e}$ since within the parabolic band approximation we neglect the ellipticity of the e pockets. The same expressions of Eq. (29) hold for the $Y$ pocket once it replaced $\Sigma_{y z}^{X} \rightarrow \Sigma_{x z}^{Y}$ and $k_{x} \rightarrow k_{y}$. Thus also the $X / Y$ pockets contribute with the opposite sign to the overall dc conductivity. By defining the real and imaginary part of the e-pocket nematic order parameter $\left(\Phi^{e}, \Delta \Gamma^{e}\right)$ :

$$
\Phi^{e} \equiv \frac{\operatorname{Re} \Sigma_{y z}^{X}-\operatorname{Re} \Sigma_{x z}^{Y}}{2}, \quad \Delta \Gamma^{e}=\frac{\left|\operatorname{Im} \Sigma_{y z}^{X}\right|-\left|\operatorname{Im} \Sigma_{x z}^{Y}\right|}{2},
$$

we can write the electronic dc conductivity anisotropy $\Delta \sigma^{e} \equiv$ $\Delta \sigma^{X}+\Delta \sigma^{Y}$ as

$$
\Delta \sigma^{e}=\sigma^{e}\left(-\frac{\Phi^{e}}{\epsilon_{0}^{e}}+\frac{\Delta \Gamma^{e}}{\Gamma_{0}^{e}}\right) .
$$

Also for the e pockets we find that the renormalized velocity and the scattering rate contribute with opposite sign to the dc conductivity anisotropy. The balance between the two effects is controlled by the nematic order parameters normalized to the Fermi energy and isotropic scattering rate, respectively, i.e., $\Phi^{e} / \epsilon_{0}^{e}$ vs $\Delta \Gamma^{e} / \Gamma_{0}^{e}$.

Summarizing, we computed analytically the anisotropy of the dc conductivity of the various pockets using the parabolic-band approximation. We find for all the pockets that the anisotropy is given by a contribution $O\left(\operatorname{Re} \Sigma^{l}\right)$ and another $O\left(\operatorname{Im} \Sigma^{l}\right)$, opposite in sign with respect to each other, whose relevance is controlled by the values of $\Phi^{h / e} / \epsilon_{0}^{h / e}$ vs $\Delta \Gamma^{h / e} / \Gamma_{0}^{h / e}$. Summing up the $\mathrm{h}$ and e pockets $\Delta \sigma^{h / e}$ we find that the sign of the anisotropy of the total dc conductivity depends on which pocket contributes more to the total conductivity and on which effect, among the scattering rate and velocity renormalization, dominates. Within the perfectly nested parabolic band approximation, in which the two $h$ pockets are equivalent, their anisotropic contributions Eq. (28) are opposite in sign and cancel out, so that the dc anisotropy is determined only by the e pockets. In this situation the overall sign of $\Delta \sigma$ depends on which effect dominates in $\Delta \sigma^{e}$, Eq. (31), i.e., the anisotropy of the velocity or the one of the scattering rate.

In real IBS systems, however, we need to account for the presence of the spin-orbit interaction that splits the $h$ pockets at $\Gamma$ and mixes their orbital content at the FS already in the tetragonal phase. Moreover, the parabolic band approximation is particularly inaccurate for the e pockets that are strongly elliptical in all IBS. Furthermore, especially for FeSe, the nematic self-energy components $\Sigma_{3}^{l}$ are not small [16], thus the expansion of the renormalized energy in $\Sigma_{3}^{l}$ performed above is not longer justified. For realistic cases then, we cannot use analytical expressions as Eqs. (23) and (24) and we need to compute the dc conductivity from Eq. (21) using a numerical estimate of the velocity and scattering rate from Eqs. (11)-(13) and (20).

\section{B. Beyond the analytical approach}

We perform a numerical estimate of the conductivity anisotropy using realistic parameters for 122 and FeSe systems in order to assess the limits of validity of the analytical 

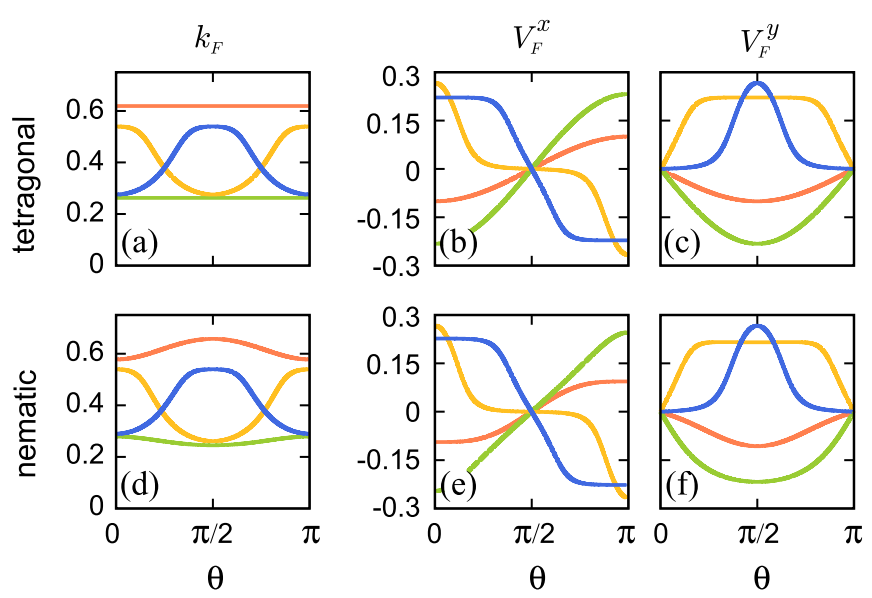

$\Gamma_{+}$

$\Gamma-$

$\mathrm{Y}$

FIG. 2. Numerical computation of FS wave vectors and velocity components for 122 system parameters in the tetragonal and nematic phase. $\Phi_{h}=\Phi_{e}=4 \mathrm{meV}$, the spin-orbit interaction is $5 \mathrm{meV}$, other band parameters are detailed in Appendix C. The $k_{F}$ are measured in units $1 / a \sim 0.375 \AA$, where $a=a_{\mathrm{FeFe}}$ is the lattice constant of the 1-Fe unit cell. The velocities are in $\mathrm{eV}$.

expressions (28) and (31) and qualitatively discuss our results in the context of the experimental outcomes found for 122 pnictides and FeSe. We assume for both 122 and FeSe equivalent band-structure parameters that result in the tetragonal FS shown in Fig. 1. The FS topology of FeSe with just the outer $h$ pocket crossing the Fermi level at $\Gamma$ already in the tetragonal phase is achieved in the calculation using a larger value of the spin-orbit interaction as well as larger values of the real part of the self-energy renormalizations in agreement with previous analysis [16]. The numerical values of the parameters used in the following are detailed in Appendix C.

a. 122 pnictides. In Fig. 2 we show for each pocket the FS wave vectors and velocities along $x / y$. To better appreciate the changes induced by the nematic order, we plot in the first row the results for the tetragonal phase and in the second ones the results obtained in the nematic phase assuming $\Phi^{h}=\Phi^{e}=4 \mathrm{meV}$. The h pockets, circular in the tetragonal phase, are weakly deformed in the nematic phase due to the small nematic order that also makes the $X / Y$ pockets slightly different [Figs. 2(a)-2(d)]. The changes in the velocities for the $\Gamma_{ \pm}$pockets appear to be quite small and do not follow monotonously the renormalization of the Fermi vectors as one could have expected [Figs. 2(e) and 2(f)]. This is in agreement with the analytical calculation outlined above, where we found that the renormalization in the velocities due to the orbital mixing and the one coming from the Fermi vector renormalization are opposite in sign, reducing the overall anisotropic effect on the velocity [see Eq. (23)]. Due to the ellipticity of the FS, the e pockets have anisotropic velocities already in the tetragonal phase [Figs. 2(b) and 2(c)] with the $X / Y$ pockets showing larger velocity along $y / x$. No qualitatively changes are visible in the nematic phase [Figs. 2(e) and 2(f)]. In Fig. 3 we show for each pocket the scattering rate obtained from Eq. (13). In the three panels we show the tetragonal value $\Gamma_{0}$, the nematic one $\Gamma_{R}$, and their difference. We find again a good agreement
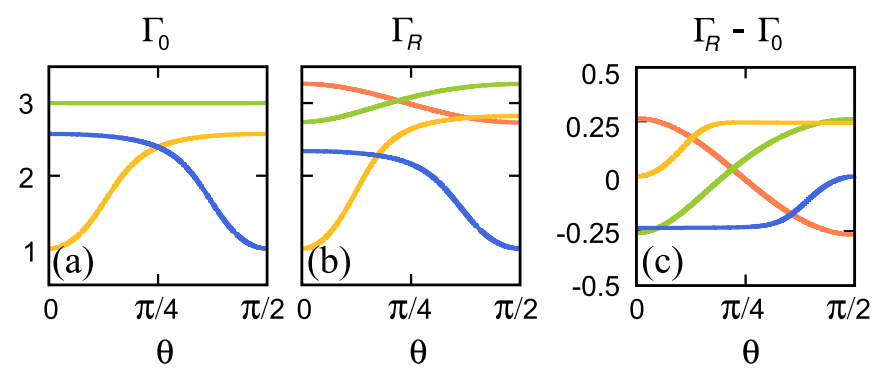

FIG. 3. Renormalized scattering rate (in meV) computed using realistic 122 system parameters. Here $\Gamma_{0}^{h}=3 \mathrm{meV}$ and $\Gamma_{0}^{e}=2 \mathrm{meV}$. We fix $\Delta \Gamma^{h}$ and $\Delta \Gamma^{e}$ considering the imaginary part of the selfenergy for each pocket changing proportionally to the real part in the nematic phase (Appendix C).

between the analytical calculations and the numerical results for the $\mathrm{h}$ pockets. As in Eq. (24), the angular dependence of the correction $\Gamma_{R}^{\Gamma_{ \pm}}-\Gamma_{0}^{\Gamma_{ \pm}}$goes almost as a $\cos 2 \theta$, even if the weak ellipticity of the h-FS induced by the nematic order causes minor deviations, e.g., the correction vanishes for the $\Gamma_{+} / \Gamma_{-}$slightly before/after $\pi / 4$. No renormalizations are found along $x / y$ for the $X / Y$ pockets since, within our model, no scattering is allowed in the $x y$ channel (Fig. 1). The location of the so-called cold spots, i.e., the position of the minima of the scattering rate for both $\mathrm{h}$ and e pockets, does not change once a realistic FS is considered and corresponds to the ones shown in Fig. 1.

We can disentangle the effect of the velocity and of the scattering rate on the dc anisotropy by computing Eq. (21) using a constant scattering rate. This result just accounts for the anisotropic effects coming from the velocity so we will refer to it as $\Delta \sigma_{V}$. In Fig. 4 we show for each pocket $\Delta \sigma_{V}$ [Figs. 4(a)-4(c)] and the complete conductivity anisotropy $\Delta \sigma$ [Figs. 4(d)-4(f)] as a function of $\Phi^{h / e}$. To easily compare
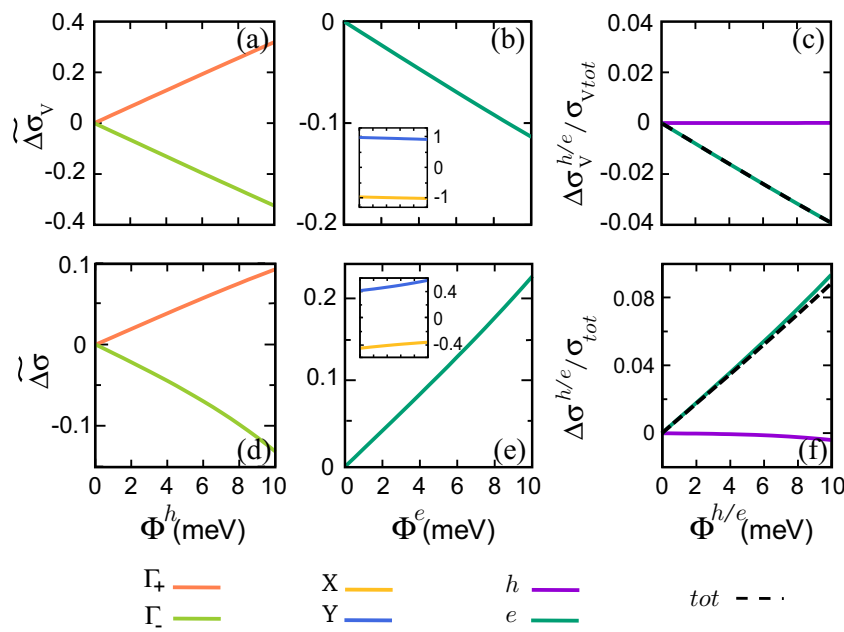

tot --

FIG. 4. Numerical computation of the velocity contribution to the de conductivity anisotropy $\Delta \sigma_{V}$ and of the total de conductivity anisotropy $\Delta \sigma$ for realistic parameter for 122. In (a) and (b) we renormalized the pocket contributions to their value in the tetragonal phase, i.e., $\Delta \sigma_{V}^{l_{ \pm}}\left(\Phi^{h / e}=0\right)$ and analogously in (d) and (e). In (c) and (f) instead we renormalize the $\mathrm{h}$ and e contributions to the total tetragonal values, i.e., $\sigma_{V \text { tot }}=\sigma_{V}\left(\Phi^{h / e}=0\right)$ and $\sigma_{\text {tot }}=\sigma\left(\Phi^{h / e}=0\right)$. 
the results of the numerics with the analytical estimate of Eqs. (28) and (31) we renormalized the $h_{ \pm} / e$ pocket anisotropy in Figs. 4(a) and 4(b) and Figs. 4(d) and 4(e) to their value in the tetragonal phase. In Figs. 4(c) and 4(f) we renormalize instead the $\mathrm{h}$ and e anisotropy to the total values of $\sigma_{V}$ and $\sigma$ obtained summing all the pockets contributions in the tetragonal phase. From the analysis of $\Delta \sigma_{V}$ we find that the sign of the anisotropic contribution proportional to $\Phi^{h / e}$ found in Eqs. (28) and (31) is robust, with the $\Gamma_{+/-}$ and the $Y / X$ pockets contributing with positive/negative terms to the dc-conductivity anisotropy [see Figs. 4(a), 4(b) and the 4(b) inset]. The h-pockets anisotropy due to the velocity [Figs. 4(a)-4(c)] is opposite in sign and grows as $\Phi^{h} / \epsilon_{0}^{h}$ in agreement with the analytical expectation. Even if the $\Gamma_{ \pm}$are not longer equivalent due to a small spin-orbit interaction, their anisotropic contributions almost cancel out so that the negative anisotropy of the e pocket is the one that determines the final results. Once the effect of the scattering rate is included in the calculation we see in Fig. 4(d) a reduction of the conductivity anisotropy for the h pocket that however still sums up to an anisotropic conductivity term close to zero [Fig. 4(f)]. In contrast, a change of sign in the overall electronic term is observed due to the larger positive contribution $\Delta \sigma^{Y}$ of the $Y$ pocket once the anisotropic scattering rate is correctly taken into account. For the set of parameters used, we find a final $\Delta \sigma>0$. The result comes from the change in the relative weight of the contribution of the $X$ and $Y$ pockets in the e term due to the different scattering rate $\Gamma_{R}^{X / Y}$. The final outcome is thus particularly sensitive to the $\Gamma_{0}^{e}$ and $\Delta \Gamma^{e}$ used and could be strongly affected by any mechanism (temperature, disorder, interactions, etc.) affecting their absolute values.

$b$. FeSe. We repeat the numerical analysis considering the case of FeSe. In Fig. 5 we show the pockets FS wave vectors and velocities both in the tetragonal and in the nematic phase assuming $\Phi^{h}=\Phi^{e}=15 \mathrm{meV}$.

With respect to the previous case, here we clearly see that the outer $\mathrm{h}$ pocket, the only one crossing the Fermi
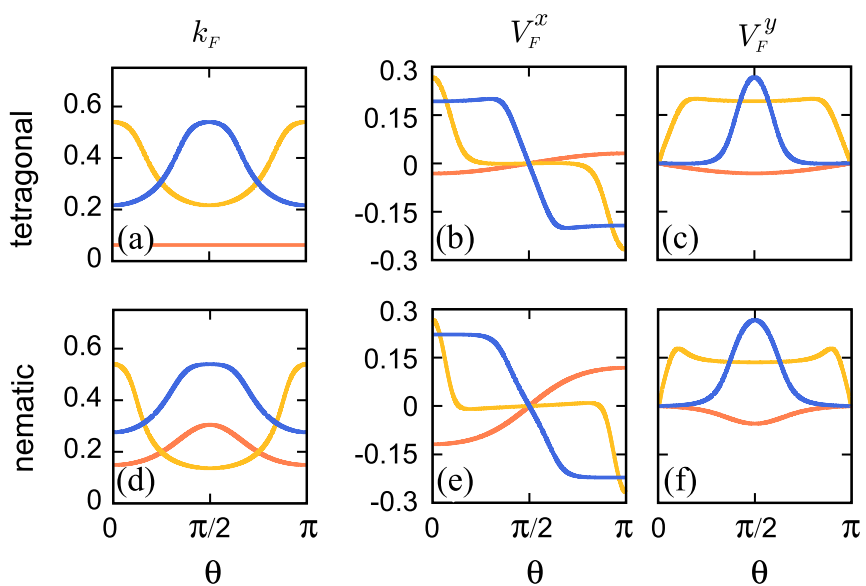

$\Gamma_{+}$

$$
\mathrm{X}
$$

Y

FIG. 5. Numerical computation of the tetragonal and nematic FS and Fermi velocity components for FeSe parameters. $\Phi_{h}=$ $\Phi_{e}=15 \mathrm{meV}$, the spin-orbit interaction is $20 \mathrm{meV}$, the other band parameters are detailed in Appendix C.
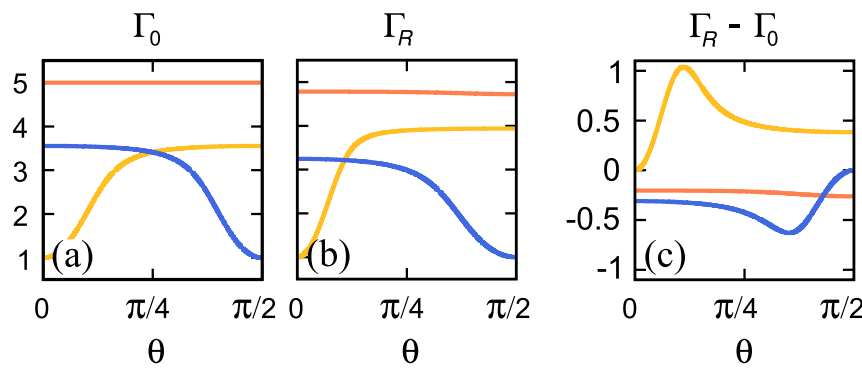

FIG. 6. Renormalized scattering rate for FeSe. $\Gamma_{0}^{h}=5 \mathrm{meV}$ and $\Gamma_{0}^{e}=2.5 \mathrm{meV} . \Delta \Gamma^{h}$ and $\Delta \Gamma^{e}$ are considered as proportional to the nematic variation of the real parts (Appendix C).

level, becomes strongly elliptical in the nematic phase due to the large nematic order which also makes the $X / Y$ pockets consistently different in size [Figs. 5(a)-5(d)]. The changes in the velocities [Figs. 5(e) and 5(f)] are similar to the ones observed for the 122 case but quantitatively more pronounced here due to the larger value of the nematic order parameters. The scattering rates for all the pockets are shown in Fig. 6. For all the pockets we find a clear deviation of the renormalized scattering rate from the analytical estimate. In particular, the angular dependence of the $\Gamma_{+}$scattering rate is very weak and does not resemble the $\cos 2 \theta$ predicted by Eq. (24). This is a consequence of the FS nematic reconstruction of FeSe. In fact, the nematic order not only makes the $\Gamma_{+}$pocket elliptical but also affects its orbital content that becomes almost completely $x z$ at the Fermi level $[16,54,55]$. As a consequence, the cold spots of the outer pocket shown in Fig. 1 do not represent anymore a minimum of the scattering since the $\Gamma_{+}$FS is mostly $x z$ also at $\theta=0$.

We study also in this case for each pocket the behavior of $\Delta \sigma_{V}$ and $\Delta \sigma$ as a function of $\Phi^{h / e}$, Fig. 7. We use the same renormalizations used in Fig. 4. The analysis of the velocity contribution reveals that the sign of the $\Phi^{h / e}$ terms of Eqs. (28) and (31) is robust also in this case. We are no
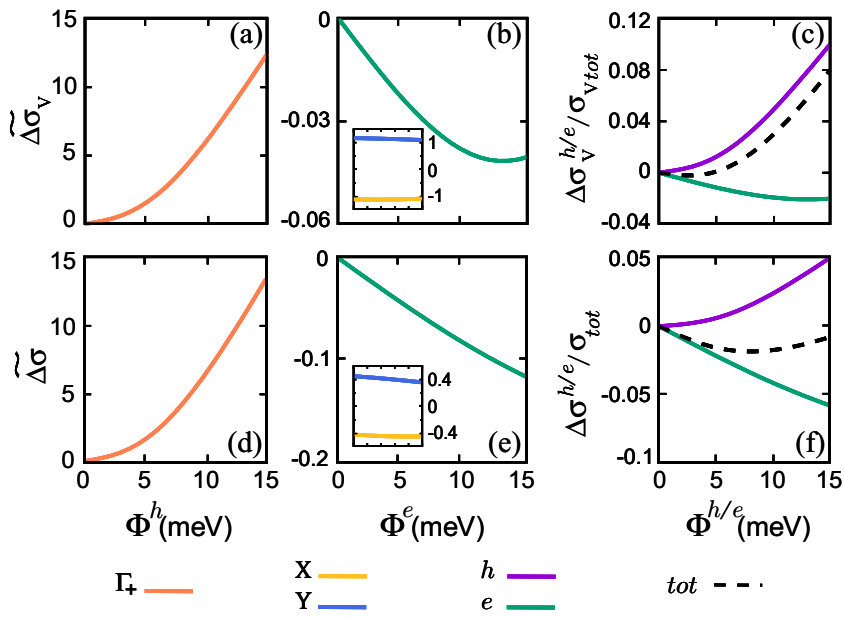

tot -- .

FIG. 7. Numerical computation of the velocity contribution to the $\mathrm{dc}$ conductivity anisotropy and of the total dc conductivity anisotropy for a realistic parameter for FeSe. All the pockets show consistent deviations of the dc anisotropy with respect the analytical expectations Eqs. (28) and (31). 
longer in the perturbative regime as one can see from the nonlinear behavior of $\Delta \sigma_{V}^{h_{+}}$, shown in Fig. 7(a), where the $\Gamma_{+}$ pocket anisotropy contribution grows much faster than what is expected from the linear dependence in Eq. (28). The final anisotropy of $\Delta \sigma_{V}$ is the result of the competition between the $\mathrm{h}$ and e terms. The inclusion of the scattering rate in the calculation strongly affects the e-pockets contribution while leaving $\Delta \sigma^{h_{+}}$almost unchanged. As a matter of fact, the scattering rate of the outer h-pocket $\Gamma_{R}^{\Gamma_{+}}$is almost isotropic, see Fig. 6, so that the anisotropic velocity is the only factor which contributes to the dc anisotropy of the $\Gamma_{+}$pocket. The final result for the dc conductivity strongly depends on the set of $\Gamma_{0}^{h / e}$ and $\Delta \Gamma^{h / e}$ used. In Figs. 7(d)-7(f) we show a case in which the inclusion of the scattering rate enhances the relative weight of the e contribution with respect the $h$ term, so that overall the negative anisotropy of the e part, due to the $X$ pocket, determines the final results shown in Fig. 7(f).

c. High-energy renormalization and nesting. An effect neglected in the above calculation is the renormalization of the quasiparticle due to local electronic interactions. It is well established that in IBS the high-energy renormalizations of the quasiparticle $Z_{\text {orb }}$ coming from local interactions are quite strong and orbital dependent. This effect, usually named orbital-selective renormalization in the literature [52], should not be confused with the effects discussed in the present work, where the orbital selectivity refers to the spin fluctuations, which affect the various orbital in a different way in the nematic phase. The high-energy renormalizations have noticeable effects on the optical conductivity in the tetragonal phase of IBS, as discussed in Ref. [58] and should be included in the above calculation. We then repeated the numerical analysis including phenomenologically the orbital renormalizations into the coherence factors $(u / v)_{R}^{l}$ entering in Eq. (19). This allows us to estimate the effects of the highenergy renormalization on the velocity contributions to the dc conductivity $\Delta \sigma_{V}$. As expected, the inclusion of a severe reduction of the coherence of the $x y$ orbital $\left(Z_{x y} \sim 0.3\right)$, which is the most correlated orbital in all IBS, leads to the suppression of the $V_{X / Y}^{x / y}$ contributions enhancing the dc anisotropy in the e pockets. Moreover, the small differentiation $(\sim 10 \%)$ of the quasiparticle masses for the $x z / y z$ orbitals in the nematic phase [59] contributes to enhance the differentiation of the $\Gamma_{ \pm}$and $X / Y$ pockets. However, the sign of the velocity contribution to the dc anisotropy is robust within the set of $Z_{\text {orb }}$ considered. The quasiparticle renormalizations affect also the conductivity via the renormalized scattering rate, however their relevance strongly depends on the set of parameters used, whose analysis goes beyond the scope of the present work.

Finally, it is worth noting that the analysis presented here, as well as the one carried out previously [16] within a momentum-independent Eliashberg scheme, does not include the physics of the band-nesting effects, which are the only ones to determine the location of the hot spots in the bandbased description [25,38]. In particular, the ellipticity of the $X / Y$ pockets suggests that the scattering rate is maximum at the location where the nesting with the $h$ pockets is realized. Within the OSSF both the orbital character and the degree of band nesting will contribute to the hot-spot location. Recent multiorbital RPA calculation in the paramagnetic state support the idea that the dominant effect in determining the scattering rate is still the orbital character of the FS [60,61]. How these results evolve below $T_{S}$ within an orbital-selective spin-nematic scenario is still an open question which certainly deserves further investigation.

\section{Comparison with experiments}

In the previous section we computed numerically the dc anisotropy for realistic parameters of 122 pnictides and FeSe. The values of the band structure parameters and self-energies used in the calculations quantitatively reproduce the main features of the FS, including the FS shrinking and the orbital FS reconstruction experimentally observed in the nematic phase of 122 and FeSe. In 122, where the nematic order parameters $\Phi^{h / e}$ are small, the h-pockets contribution to the dc conductivity anisotropy is well approximated by the analytical estimate Eq. (28), while we observe consistent deviations in FeSe. Nonetheless, for both 122 and FeSe systems the sign of the anisotropic contribution coming from the renormalized velocity $\Delta \sigma_{V}$ is robust. In both cases considered in Sec. III B we managed to match the experimental result $\Delta \sigma_{\mathrm{dc}}(\mathrm{FeSe})<0$ and $\Delta \sigma_{\mathrm{dc}}(122)>0$, once the renormalization of the scattering rate is included in the calculation. As already mentioned, the final result is still somehow sensitive to the set of parameters used. Thus, in this last section we discuss in general which are the possibilities to match the experimental results regardless the precise choice of parameters used in Sec. III B.

Concerning 122 systems, as long as the h-pocket contributions to the dc anisotropy cancel out, the final result is controlled by the e pockets. Since they have a strong elliptical deformation, their overall contribution to the dc anisotropy cannot be predicted from the analytical result Eq. (31), and the final outcome depends on the relative weight of the $X$ and $Y$ pockets and on the relevance of the scattering-rate anisotropy over the contribution $\Delta \sigma_{V}^{e}$. Even in doped 122 compounds the h-pocket contributions cancellation still occurs since the relatively small value of the spin-orbit splitting at $\Gamma$ guarantees that the Fermi energy is the same for the h pockets. However, doping changes both the size of the pockets and the degree of nesting between $h$ and e pockets. Both effects contribute to change the relative weight of the $X / Y$ e pockets as well as the balance between the velocity vs scattering-rate anisotropic contributions and can be at the origin of the different sign of $\Delta \sigma_{\mathrm{dc}}$ experimentally observed between the h- and e-doped side of the 122 phase diagram.

For what concerns FeSe, the presence of a single h pocket and its strong orbital reconstruction lead to rather different physics. In particular, since the nematic FS reconstruction makes the whole $\Gamma_{+}$FS mostly $x z$ even at $\theta=0$, the expected anisotropy of the renormalized scattering rate is absent, see Fig. 6. As a consequence the $\Gamma_{+}$anisotropic contribution is more likely controlled by the velocity anisotropy. This result should be contrasted with the outcomes of Ref. [47], where the difference between 122 and FeSe is fully ascribed to a different behavior of the scattering-rate anisotropy in the two compounds. In our picture the FeSe dc-conductivity 
anisotropy emerges from a subtle interplay between the competing effects coming from the scattering rate and the velocity, as it has been recently suggested by optical conductivity experiments in FeSe [62]. It is worth noting that recent ARPES experiments reveal a strong $k_{z}$ dependence of the orbital composition of the $\Gamma_{+}$FS [53], with the FS a $k_{z}=\pi$ recovering $y z$ character at $\theta=0$. As a consequence, also the scattering rate anisotropy on $\Gamma_{+}$is expected to be larger at $k_{z}=\pi$ and its effect on the dc-conductivity anisotropy can possibly compete with the velocity term at this $k_{z}$. This observation calls for a more complete analysis of the dc anisotropy involving also the $k_{z}$ dependence of the FS.

\section{CONCLUSIONS}

In conclusion, we computed the dc-conductivity anisotropy in the nematic phase of IBS using the orbital-selective spinnematic scenario that accounts for the orbital content of the FS $[19,48]$. In this scenario the dc anisotropy of the nematic phase of IBS depends on the scattering rate and velocity renormalizations due to self-energy corrections. Both scattering rate and velocity are affected by the FS nematic reconstruction. The scattering rate is strongly affected by the orbital content of the FS, and the location of its minima on the FS is found in correspondence of the less renormalized orbitals giving rise to cold spots. The velocity renormalization is sensitive both to the orbital mixing and to the shrinking of the FS induced by the nematic order, with the former effect dominating over the latter. Due to this effect we find the unexpected result that the conductivity increases in the direction in which the self-energy is larger and the shrinking is stronger. For both $\mathrm{h}$ and e carriers the contribution of the velocity to the dc anisotropy is opposite in sign to the one of the scattering rate. This is in agreement with recent optical conductivity experiment in FeSe [62] where it is shown that scattering rate and velocity contribute to the conductivity anisotropy with opposite signs. Our results naturally follow from the spin-orbital entanglement implicit in the OSSF model and are new results in contrast to the band spin-nematic scenario $[2,3]$. In particular we demonstrated that the usual expectation of anisotropic magnetic fluctuations giving rise only to an anisotropy in the inelastic scattering rate [38] is not longer valid once the orbital degree of freedom is taken into account in the theoretical description.

We performed numerical calculation for representative parameters for 122 pnictides and FeSe. We verified that for the 122 system the analytical estimate represents a good approximation of the numerical with the overall h-pockets contribution vanishing even once a finite spin-orbit splitting at $\Gamma$ is considered. Numerical results for FeSe instead deviate from the analytical expectations due to the huge nematic FS reconstruction. We also discuss how the conductivity anisotropy depends on the system parameters. It can be dominated by either electron or hole pocket conductivity and depends on ellipticity and high-energy renormalizations. The OSSF scenario provides then a suitable framework where the same mechanism due to orbital-spin interplay can reconcile the experimental observations reported in different families of iron-based superconductors.

\section{ACKNOWLEDGMENTS}

We acknowledge L. Degiorgi for useful discussions. L.B. acknowledges financial support by Italian MAECI under the collaborative Italia-India project SuperTop-PGR04879. B.V. acknowledges funding from MINECO (Spain) via Grant No. FIS2014-53219-P and Fundación Ramón Areces. L.B., L.F., and B.V. acknowledge the cost action Nanocohybri CA16218.

\section{APPENDIX A: GREEN'S FUNCTION AND PERFECTLY NESTED PARABOLIC BAND APPROXIMATION}

In this Appendix we calculate the dressed Green's function to be used in the dc conductivity. We also detail the perfect nested parabolic band approximation to perform the analytical calculation of the conductivity.

\section{Green's function}

The bare Hamiltonian consists of a four-pocket model with two h pockets at $\Gamma, \Gamma_{ \pm}$and two e pockets at $X$ and $Y$ adapted from the low-energy model considered in Ref. [57]. Each pocket is described using a spinor representation in the pseudo-orbital space:

$$
H_{0}^{l}=\sum_{\mathbf{k}, \sigma} \psi_{\mathbf{k} \sigma}^{\dagger l} \hat{H}_{0 \mathbf{k}}^{l} \psi_{\mathbf{k} \sigma}^{l},
$$

where $l=\Gamma, X, Y$ and the spinors are defined as $\psi_{\mathbf{k} \sigma}^{\Gamma}=$ $\left(c_{\mathbf{k}, \sigma}^{y z}, c_{\mathbf{k} \sigma}^{x z}\right)$ and $\psi_{\mathbf{k} \sigma}^{X / Y}=\left(c_{\mathbf{k} \sigma}^{y z / x z}, c_{\mathbf{k} \sigma}^{x y}\right)$. The matrix $\hat{H}_{0 \mathbf{k}}^{l}$ has the general form

$$
\hat{H}_{0 \mathbf{k}}^{l}=h_{0}^{l} \hat{\tau}_{0}+\vec{h}^{l} \cdot \overrightarrow{\hat{\tau}}=\left(\begin{array}{cc}
h_{0}^{l}+h_{3}^{l} & h_{1}^{l}-i h_{2}^{l} \\
h_{1}^{l}+i h_{2}^{l} & h_{0}^{l}-h_{3}^{l}
\end{array}\right),
$$

with $\hat{\tau}$ matrices representing the pseudo-orbital spin. The $h^{\Gamma}$ components read as

$$
\begin{aligned}
& h_{0}^{\Gamma}=\epsilon^{\Gamma}-a^{\Gamma} \mathbf{k}^{2}, \\
& h_{1}^{\Gamma}=-2 b^{\Gamma} k_{x} k_{y}, \\
& h_{3}^{\Gamma}=b^{\Gamma}\left(k_{x}^{2}-k_{y}^{2}\right),
\end{aligned}
$$

and for the $X$ pocket,

$$
\begin{aligned}
& h_{0}^{X}=\left(h^{y z}+h^{x y}\right) / 2, \\
& h_{2}^{X}=v k_{y}, \\
& h_{3}^{X}=\left(h^{y z}-h^{x y}\right) / 2-b\left(k_{x}^{2}-k_{y}^{2}\right),
\end{aligned}
$$

where $h^{y z}=-\epsilon^{y z}+a^{y z} \mathbf{k}^{2}$ and $h^{x y}=-\epsilon^{x y}+a^{x y} \mathbf{k}^{2}$. Analogous expressions hold for the $Y$ pocket provided that one exchange $k_{x}$ by $k_{y}$. Rotating the Hamiltonian $\hat{H}_{0 \mathbf{k}}^{l}$ into the band basis we have $\hat{H}_{0 \mathbf{k}}^{l}=\hat{\mathcal{U}}^{l^{-1}} \hat{\Lambda}^{l} \hat{\mathcal{U}}^{l}$ where $\hat{\Lambda}^{l}=\operatorname{diag}\left(E_{\mathbf{k}}^{l+}, E_{\mathbf{k}}^{l-}\right)$ is the eigenvalue matrix and $E_{\mathbf{k}}^{l \pm}$ is given by

$$
E_{\mathbf{k}}^{l \pm}=h_{0}^{l} \pm h^{l}=h_{0}^{l} \pm \sqrt{\left(h_{1}^{l}\right)^{2}+\left(h_{2}^{l}\right)^{2}+\left(h_{3}^{l}\right)^{2}} .
$$

The fermionic band operators $c^{l \pm}$ from $H_{0}^{l}=$ $\sum_{\mathbf{k}, \sigma} E_{\mathbf{k}}^{l \pm} c_{\mathbf{k} \sigma}^{\dagger l \pm} c_{\mathbf{k} \sigma}^{l \pm}$ are obtained rotating the orbital spinors via $\hat{\mathcal{U}}^{l}$. The unitary $\hat{\mathcal{U}}^{l}$ matrix has the common form:

$$
\hat{\mathcal{U}}^{l}=\left(\begin{array}{cc}
u^{l} & -v^{l} \\
v^{* l} & u^{* l}
\end{array}\right)
$$


where we drop the momentum and spin indices for simplicity. Explicitly for the h pockets at $\Gamma, c^{\Gamma \pm} \equiv h^{ \pm}$we have

$$
\left(\begin{array}{l}
h^{+} \\
h^{-}
\end{array}\right)=\left(\begin{array}{cc}
u^{\Gamma} & -v^{\Gamma} \\
v^{* \Gamma} & u^{* \Gamma}
\end{array}\right)\left(\begin{array}{l}
c^{y z} \\
c^{x z}
\end{array}\right)=\hat{\mathcal{U}}^{\Gamma}\left(\begin{array}{l}
c^{y z} \\
c^{x z}
\end{array}\right) .
$$

Analogous expressions hold for the $X / Y$ e-pockets fermionic operators $c^{X / Y \pm} \equiv e^{X / Y \pm}$, provided that the corresponding orbital spinors $\psi^{X / Y}=\left(c^{y z / x z}, c^{x y}\right)$ are used. The coherence factors are

$$
\begin{aligned}
u^{l} & =\frac{1}{\sqrt{2}} \sqrt{1+\frac{h_{3}^{l}}{h^{l}}}, \\
v^{* l} & =\frac{1}{\sqrt{2}} \frac{h_{1}^{l}+i h_{2}^{l}}{\sqrt{\left(h_{1}^{l}\right)^{2}+\left(h_{2}^{l}\right)^{2}}} \sqrt{1-\frac{h_{3}^{l}}{h^{l}}} .
\end{aligned}
$$

Knowing the dispersion relation and the coherence factors inside the rotation matrix $\hat{\mathcal{U}}^{l}$ we can obtain the bare pocket Green's functions. Since $\hat{G}_{0}^{l^{-1}}=\omega \hat{\mathbb{1}}-\hat{H}_{0 \mathbf{k}}^{l}$ simply $\hat{G}_{0}^{l^{-1}}=$ $\hat{\mathcal{U}}^{l^{-1}}\left(\omega \hat{\mathbb{1}}-\hat{\Lambda}^{l}\right) \hat{\mathcal{U}}^{l}$ with $\hat{\Lambda}^{l}=\operatorname{diag}\left(E^{l_{+}}, E^{l_{-}}\right)$.

We now turn our attention to the OSSF self-energy. The low-energy interacting Hamiltonian describing the spin exchange between $h$ and e pockets is given in Eq. (4). The corresponding Dyson equation for each pocket is

$$
\hat{G}^{l^{-1}}(\mathbf{k}, \omega)=\hat{G}_{0}^{l^{-1}}(\mathbf{k}, \omega)-\hat{\Sigma}^{l}(\omega),
$$

where $\quad \hat{G}_{0}^{l^{-1}}(\mathbf{k}, \omega)=\omega \hat{\mathbb{1}}-\hat{H}_{0 \mathbf{k}}^{l} \quad$ and $\quad \hat{\Sigma}^{l}(\omega)=\Sigma_{0}^{l}(\omega) \hat{\tau}_{0}+$ $\Sigma_{3}^{l}(\omega) \hat{\tau}_{3}$ is the self-energy due to the OSSF defined in the main text. From now on we do not display the $(\mathbf{k}, \omega)$ dependence. We can define the renormalized Hamiltonian as $\hat{H}^{l}=\hat{H}_{0}^{l}+\hat{\Sigma}^{l}$. Rotating the Hamiltonian $\hat{H}^{l}$ into the band basis we have $\hat{H}^{l}=\hat{\mathcal{U}}_{R}^{l^{-1}} \hat{\Lambda}_{R}^{l} \hat{\mathcal{U}}_{R}^{l}$ where $\hat{\Lambda}_{R}^{l}=\operatorname{diag}\left(E_{R}^{l_{+}}, E_{R}^{l_{-}}\right)$is the eigenvalue matrix and $E_{R}^{l_{ \pm}}$is given by

$$
\begin{aligned}
E_{R}^{l_{ \pm}}= & h_{0}^{l}+\Sigma_{0}^{l} \pm h_{R}^{l}=h_{0}^{l}+\Sigma_{0}^{l} \\
& \pm \sqrt{\left(h_{1}^{l}\right)^{2}+\left(h_{2}^{l}\right)^{2}+\left(h_{3}^{l}+\Sigma_{3}^{l}\right)^{2}} .
\end{aligned}
$$

Since the self-energy given in Eq. (A9) is complex, we define the renormalized energy dispersion relation as $\epsilon_{R}^{l_{ \pm}}=\operatorname{Re} E_{R}^{l_{ \pm}}$ and the renormalized scattering rate as $\Gamma_{R}^{l_{ \pm}}=\delta \Gamma+\left|\operatorname{Im} E_{R}^{l_{ \pm}}\right|$ with $\delta \Gamma$ some scattering background. The unitary $\hat{\mathcal{U}}_{R}^{l}$ matrix has a structure analogous to Eq. (A6) provided that the coherence factors are given by

$$
\begin{aligned}
& u_{R}^{l}=\frac{1}{\sqrt{2}} \sqrt{1+\frac{h_{3}^{l}+\Sigma_{3}^{l}}{h_{R}^{l}}}, \\
& v_{R}^{* l}=\frac{1}{\sqrt{2}} \frac{h_{1}^{l}+i h_{2}^{l}}{\sqrt{\left(h_{1}^{l}\right)^{2}+\left(h_{2}^{l}\right)^{2}}} \sqrt{1-\frac{h_{3}^{l}+\Sigma_{3}^{l}}{h_{R}^{l}}} .
\end{aligned}
$$

We can take into account in our model the presence of a finite spin-orbit interaction by replacing $h_{R}^{\Gamma} \rightarrow h_{R, \mathrm{SO}}^{\Gamma}=$ $\sqrt{\left(h_{1}^{\Gamma}\right)^{2}+\left(h_{3}^{\Gamma}+\Sigma_{3}^{\Gamma}\right)^{2}+\left(\lambda^{2} / 4\right)}$. This affects both the $E_{R}^{\Gamma_{ \pm}}$ and the coherence factors $(u / v)_{R}^{\Gamma}$.

\section{Perfectly nested circular Fermi surfaces model}

For the analytical approach, we write the bare pocket Hamiltonian Eq. (A2) in polar coordinates with $\theta=$ $\arctan k_{y} / k_{x}$, for the hole

$$
\begin{aligned}
& h_{0}^{\Gamma}=\epsilon^{\Gamma}-a k^{2}, \\
& h_{1}^{\Gamma}=-b k^{2} \sin (2 \theta), \\
& h_{3}^{\Gamma}=b k^{2} \cos (2 \theta),
\end{aligned}
$$

and for the electron pockets

$$
\begin{aligned}
& h_{0}^{X / Y}=-\epsilon^{X / Y}+a k^{2}, \\
& h_{2}^{X / Y}=b k^{2} \sin (2 \theta), \\
& h_{3}^{X / Y}=\mp b k^{2} \cos (2 \theta) .
\end{aligned}
$$

For simplicity let us further assume $\epsilon^{X} \equiv \epsilon^{Y} \equiv \epsilon^{e}$. It is easy to check that the orbital content of the four-pocket model for IBS in Fig. 1 is reproduced in the parabolic approximation. Using Eq. (A8) the coherence factors take the expression

$$
\begin{aligned}
& \left|u^{\Gamma}\right|^{2}=\left|u^{Y}\right|^{2}=\left|v^{X}\right|^{2}=\cos \theta^{2}, \\
& \left|v^{\Gamma}\right|^{2}=\left|v^{Y}\right|^{2}=\left|u^{X}\right|^{2}=\sin \theta^{2} .
\end{aligned}
$$

To allow analytical treatment, we expand $E_{R}^{l_{ \pm}}$and $(u / v)_{R}^{l}$ Eqs. (A10) and (A11) up to first order in $\Sigma_{3}$ and we neglect the imaginary part of the self-energy since it goes to zero at low $\omega$ and $T=0$. The dressed coherence factors are given by

$$
\begin{aligned}
& \left|u_{R}^{l}\right|^{2}=\left|u^{l}\right|^{2}\left(1+\frac{2 \operatorname{Re} \Sigma_{3}^{l}}{h^{l}}\left|v^{l}\right|^{2}\right), \\
& \left|v_{R}^{l}\right|^{2}=\left|v^{l}\right|^{2}\left(1-\frac{2 \operatorname{Re} \Sigma_{3}^{l}}{h^{l}}\left|u^{l}\right|^{2}\right),
\end{aligned}
$$

which are the expressions quoted in Eq. (14). The dressed dispersion relations $\epsilon_{R}^{l_{ \pm}}=\operatorname{Re} E_{R}^{l_{ \pm}}$become

$$
\epsilon_{R}^{l_{ \pm}}=\epsilon^{l_{ \pm}}+\operatorname{Re} \Sigma_{0}^{l} \pm \frac{h_{3}^{l}}{h^{l}} \operatorname{Re} \Sigma_{3}^{l} .
$$

Replacing the values for the case of circular FS given in Eqs. (A12) and (A13) and calculate it at the Fermi surface we get

$$
\begin{aligned}
\epsilon_{R}^{\Gamma_{ \pm}} & =\epsilon_{0}^{h} \pm \cos 2 \theta \operatorname{Re} \Sigma_{3}^{\Gamma}, \\
\epsilon_{R}^{X / Y} & =-\epsilon_{0}^{e} \mp \cos 2 \theta \operatorname{Re} \Sigma_{3}^{X / Y},
\end{aligned}
$$

where we defined the tetragonal band energy for the $\mathrm{h}$ pockets as $\epsilon_{0}^{h}=\epsilon^{\Gamma}+\operatorname{Re} \Sigma_{0}^{\Gamma}$ and $\epsilon_{0}^{e}=\epsilon^{e}-\operatorname{Re} \Sigma_{0}^{X / Y}$ for the e pockets. The scattering rate $\Gamma_{R}^{l_{ \pm}}=\delta \Gamma+\left|\operatorname{Im} E_{R}^{l_{ \pm}}\right|$acquires the expression

$$
\Gamma_{R}^{l_{ \pm}} \approx \delta \Gamma+\left|\operatorname{Im} \Sigma_{0}^{l}\right| \pm \frac{h_{3}^{l}}{h^{l}}\left|\operatorname{Im} \Sigma_{3}^{l}\right| .
$$

Using Eqs. (A12) and (A13) we get

$$
\begin{aligned}
\Gamma_{R}^{\Gamma_{ \pm}} & =\Gamma_{0}^{h} \pm \cos 2 \theta\left|\operatorname{Im} \Sigma_{3}^{\Gamma}\right|, \\
\Gamma_{R}^{X / Y} & =\Gamma_{0}^{e} \mp \cos 2 \theta\left|\operatorname{Im} \Sigma_{3}^{X / Y}\right|,
\end{aligned}
$$

where we have separated the angular dependent renormalization $\sim \operatorname{Im} \Sigma_{3}^{l}$ from the tetragonal constant part $\Gamma_{0}^{h / e}=\delta \Gamma+$ $\left|\operatorname{Im} \Sigma_{0}^{\Gamma / e}\right|$. From Eq. (A19) we find analytically the locations 
of the cold spots on the FS where the minimum value of the scattering rate is found:

$$
\begin{aligned}
\Gamma_{R}^{\Gamma_{+}}\left(\theta=\frac{\pi}{2}\right) & =\delta \Gamma+\left|\operatorname{Im} \Sigma_{x z}^{\Gamma}\right|, \\
\Gamma_{R}^{\Gamma_{-}}(\theta=0) & =\delta \Gamma+\left|\operatorname{Im} \Sigma_{x z}^{\Gamma_{z}}\right|, \\
\Gamma_{R}^{X / Y}(\theta=0) & =\Gamma_{R}^{Y}\left(\theta=\frac{\pi}{2}\right)=\delta \Gamma .
\end{aligned}
$$

This result is easy to understand due to the OSSF shown in Fig. 1 where cold spots are also shown. In the nematic phase, $\mathrm{SF}$ with momentum $\mathbf{Q}_{X}$ are bigger than the ones with $\mathbf{Q}_{Y}$. As a consequence, the largest scattering is found in the $y z$ orbital due to the orbital-selective nature of SF, while the $x y$-orbital component is not renormalized given the absence of $x y$-OSSF.

\section{APPENDIX B: COMPUTATION OF THE DC CONDUCTIVITY AND ANALYTICAL RESULTS FOR THE PERFECTLY NESTED CIRCULAR FS}

We calculate the dc conductivity in the bubble approximation. The self-energy corrections computed within an Eliashberg-like treatment are momentum independent so that vertex corrections vanish identically. The dc conductivity is given by

$$
\operatorname{Re} \sigma_{\alpha}(\Omega)=-\frac{e^{2}}{V} \frac{\operatorname{Im} \Pi_{\alpha}\left(\mathbf{q}=0, i \Omega_{m} \rightarrow \Omega\right)}{\Omega},
$$

where $\alpha=x, y, V$ is the unit-cell volume, and $\Pi_{\alpha}$ is the current-current correlation function in the bubble approximation given by

$$
\Pi_{\alpha}\left(i \Omega_{m}\right)=2 T \sum_{l \mathbf{k} n} \operatorname{Tr}\left[\hat{G}^{l}\left(\mathbf{k}, i \omega_{n}\right) \hat{V}_{\mathbf{k}_{\alpha}}^{l} \hat{G}^{l}\left(\mathbf{k}, i \omega_{n}+i \Omega_{m}\right) \hat{V}_{\mathbf{k}_{\alpha}}^{l}\right] .
$$

$\hat{V}_{\mathbf{k}_{\alpha}}^{l}=\partial_{\mathbf{k}_{\alpha}} \hat{H}_{0}^{l}$ is the bare velocity operator and $\hat{G}^{l}\left(\mathbf{k}, i \omega_{n}\right)$ is the renormalized Green's function from the orbital-selective spin fluctuations. We work in the rotated basis and replace $\hat{G}^{l}\left(\mathbf{k}, i \omega_{n}\right)=\hat{\mathcal{U}}_{R}^{l}\left(\mathbf{k}, i \omega_{n}\right)\left[i \omega_{n} \hat{\mathbb{1}}-\hat{\Lambda}_{R}^{l}(\mathbf{k}, \omega)\right]^{-1} \hat{\mathcal{U}}_{R}^{l^{-1}}\left(\mathbf{k}, i \omega_{n}\right)$ in Eq. (B2). Since we are interested in the dc conductivity $(\Omega \rightarrow$ 0 ) the important term in the trace is

$$
\begin{aligned}
\operatorname{Tr} & \left\{\hat { \mathcal { U } } _ { R } ^ { l } ( \mathbf { k } , i \omega _ { n } ) \left[\left(i \omega_{n} \hat{\mathbb{1}}-\hat{\Lambda}_{R}^{l}(\mathbf{k}, \omega)\right]^{-1} \hat{\mathcal{U}}_{R}^{l^{-1}}\left(\mathbf{k}, i \omega_{n}\right) \hat{V}_{\mathbf{k}_{\alpha}}^{l}\right.\right. \\
& \left.\times \hat{\mathcal{U}}_{R}^{l}\left(\mathbf{k}, i \omega_{n}\right)\left[i \omega_{n} \hat{\mathbb{1}}-\hat{\Lambda}_{R}^{l}(\mathbf{k}, \omega)\right]^{-1} \hat{\mathcal{U}}_{R}^{l^{-1}}\left(\mathbf{k}, i \omega_{n}\right) \hat{V}_{\mathbf{k}_{\alpha}}^{l}\right\} .
\end{aligned}
$$

Using the cyclic property of the trace allows us to define the renormalized velocity $\hat{V}_{R \mathbf{k}_{\alpha}}^{l}=\hat{\mathcal{U}}_{R}^{l^{-1}}\left(\mathbf{k}, i \omega_{n}\right) \hat{V}_{\mathbf{k}_{\alpha}}^{l} \hat{\mathcal{U}}_{R}^{l}\left(\mathbf{k}, i \omega_{n}\right)$. Operating the trace and taking the limit $\Omega \rightarrow 0$ we get that the trace in Eq. (B3) can be written as

$$
g_{+}^{2}\left(\mathbf{k}, i \omega_{n}\right)\left(V_{R \mathbf{k}_{\alpha}}^{l_{+}}\right)^{2}+g_{-}^{2}\left(i \omega_{n}\right)\left(V_{R \mathbf{k}_{\alpha}}^{l_{-}}\right)^{2},
$$

where $V_{R \mathbf{k}_{\alpha}}^{l_{ \pm}}$are given by

$$
V_{R \mathbf{k}_{\alpha}}^{l_{ \pm}}=V_{\mathbf{k} \alpha}^{l_{11}}\left|u_{R}^{l}\right|^{2} \pm V_{\mathbf{k} \alpha}^{l_{12}} u_{R}^{* l} v_{R}^{* l} \pm V_{\mathbf{k} \alpha}^{l_{21}} u_{R}^{l} v_{R}^{l}+V_{\mathbf{k} \alpha}^{l_{22}}\left|v_{R}^{l}\right|^{2} .
$$

Here $V_{\mathbf{k} \alpha}^{l} \eta_{\eta^{\prime}}$ are the $\eta \eta^{\prime}$ component of $\hat{V}_{\mathbf{k}_{\alpha}}^{l}=\partial_{\mathbf{k}_{\alpha}} \hat{H}_{0}^{l}$ and $(u / v)_{R}^{l}$ are the renormalized coherence factors defined in Eq. (A11). The multiorbital character of the problem gives rise to selfenergy effects in the velocities via the coherence factors especially in the nematic phase. Expressing the Green's function in terms of the spectral functions $A^{l_{ \pm}}(\mathbf{k}, \omega)$ we finally arrive to the results of Eq. (17):

$$
\sigma_{\alpha}^{l_{ \pm}}=\frac{2 \pi e^{2}}{N} \sum_{\mathbf{k}} \int_{-\infty}^{\infty} d \omega\left(-\frac{\partial f(\omega)}{\partial \omega}\right)\left[V_{R \mathbf{k}_{\alpha}}^{l_{ \pm}}(\omega)\right]^{2}\left[A_{\mathbf{k}}^{l_{ \pm}}(\omega)\right]^{2},
$$

where $f(\omega)$ the Fermi distribution function. Notice that the renormalized velocities $V_{R \mathbf{k}_{\alpha}}^{l_{ \pm}}(\omega)$ depend on frequency and on the orbital self-energy through the dependence of the coherence factors on $\Sigma_{3}^{l}(\omega)$. The renormalized spectral function can be written as

$$
A_{\mathbf{k}}^{l_{ \pm}}(\omega)=\frac{1}{\pi} \frac{\Gamma_{R \mathbf{k}}^{l_{ \pm}}(\omega)}{\left[\Gamma_{R \mathbf{k}}^{l_{ \pm}}(\omega)\right]^{2}+\left[\omega-\epsilon_{R \mathbf{k}}^{l_{ \pm}}(\omega)\right]^{2}},
$$

where $\quad \epsilon_{R \mathbf{k}}^{l_{ \pm}}(\omega)=\operatorname{Re} E_{R}^{l_{ \pm}}(\mathbf{k}, \omega) \quad$ and $\quad \Gamma_{R \mathbf{k}}^{l_{ \pm}}(\omega)=\delta \Gamma+$ $\left|\operatorname{Im} E_{R}^{l_{ \pm}}(\mathbf{k}, \omega)\right|$. At low temperature $-\frac{\partial f(\omega)}{\partial \omega} \rightarrow \delta(\omega)$ selects only states at the Fermi level

$$
\sigma_{\alpha}^{l_{ \pm}}=\frac{2 \pi e^{2}}{N} \sum_{\mathbf{k}}\left(V_{R \mathbf{k}_{\alpha}}^{l_{ \pm}}\right)^{2}\left(A_{\mathbf{k}}^{l_{ \pm}}\right)^{2} .
$$

Moreover, assuming $\Gamma_{R}^{l_{ \pm}} \rightarrow 0$, the spectral function $A_{\mathbf{k}}^{l_{ \pm}}$can be approximated as

$$
\left(A_{\mathbf{k}}^{l_{ \pm}}\right)^{2} \longrightarrow \frac{1}{2 \pi \Gamma_{R \mathbf{k}}^{l_{ \pm}}} \delta\left(\epsilon_{R \mathbf{k}}^{l_{ \pm}}\right)
$$

Replacing this expression in Eq. (B7) we get that the conductivity is

$$
\begin{aligned}
\sigma_{\alpha}^{l_{ \pm}} & =\frac{e^{2}}{N} \sum_{\mathbf{k}} \frac{\left(V_{R \mathbf{k}_{\alpha}}^{l_{ \pm}}\right)^{2}}{\Gamma_{R \mathbf{k}}^{l_{ \pm}}} \delta\left(\epsilon_{R \mathbf{k}}^{l_{ \pm}}\right) \\
& =\frac{e^{2}}{N} \int \frac{d \mathbf{k}^{2}}{(2 \pi)^{2}} \frac{\left(V_{R \mathbf{k}_{\alpha}}^{l_{ \pm}}\right)^{2}}{\Gamma_{R \mathbf{k}}^{l_{ \pm}}} \frac{\delta\left(\mathbf{k}-\mathbf{k}_{F}\right)}{\left|\nabla \epsilon_{R \mathbf{k}}^{l_{ \pm}}\right|} .
\end{aligned}
$$

Within the parabolic band approximation and using the expansion up to the first order in $\Sigma_{3}^{l}$ of the renormalized energy, we can derive analytically the conductivity given in Eq. (B9). We use the expressions of the renormalized energies and scattering rates derived in Appendix A. Using the expressions Eq. (A15) for the coherence factors, it is easy to check the velocity given in Eq. (B4) can be expressed as the derivative of the renormalized dispersion relation given in Eq. (A16), so $V_{R \mathbf{k} \alpha}^{l \pm}=\partial_{k_{\alpha}} \epsilon_{R}^{l_{ \pm}}(\mathbf{k})$. Explicitly for the h pockets at $\Gamma$ we have

$$
\begin{aligned}
V_{R \mathbf{k} \alpha}^{\Gamma_{ \pm}}= & \partial_{k_{\alpha}} h_{0}^{\Gamma} \pm \partial_{k_{\alpha}} h_{1}^{\Gamma} \frac{h_{1}^{\Gamma}}{h^{\Gamma}} \pm \partial_{k_{\alpha}} h_{3}^{\Gamma} \frac{h_{3}^{\Gamma}}{h^{\Gamma}} \\
& \pm \operatorname{Re} \Sigma_{3}^{\Gamma} \frac{h_{1}^{\Gamma}}{\left(h^{\Gamma}\right)^{2}}\left[\partial_{k_{\alpha}} h_{3}^{\Gamma} \frac{h_{1}^{\Gamma}}{h^{\Gamma}}-\partial_{k_{\alpha}} h_{1}^{\Gamma} \frac{h_{3}^{\Gamma}}{h^{\Gamma}}\right]
\end{aligned}
$$

and analogous expressions for $V_{R \mathbf{k} \alpha}^{X}$ and $V_{R \mathbf{k} \alpha}^{Y}$. The first three terms in Eq. (B10) corresponds to the bare velocity, while the term multiplied by $\operatorname{Re} \Sigma_{3}^{\Gamma}$ accounts for the renormalization in the velocity due to OSSF self-energy corrections. Using the explicit definition of $h_{0}^{l}$ and $\vec{h}^{l}$, the velocities for the various 
pockets read

$$
\begin{aligned}
V_{R x}^{\Gamma_{ \pm}} & =-\frac{k \cos \theta}{m^{\Gamma_{ \pm}}} \pm 4 \operatorname{Re} \Sigma_{3}^{\Gamma} \frac{k \cos \theta \sin \theta^{2}}{k^{2}}, \\
V_{R y}^{\Gamma_{ \pm}} & =-\frac{k \sin \theta}{m^{\Gamma_{ \pm}}} \mp 4 \operatorname{Re} \Sigma_{3}^{\Gamma_{3}} \frac{k \sin \theta \cos \theta^{2}}{k^{2}}, \\
V_{R x}^{X / Y} & =\frac{k \cos \theta}{m^{X / Y}} \mp 4 \operatorname{Re} \Sigma_{3}^{X / Y} \frac{k \cos \theta \sin \theta^{2}}{k^{2}}, \\
V_{R y}^{X / Y} & =\frac{k \sin \theta}{m^{X / Y}} \pm 4 \operatorname{Re} \Sigma_{3}^{X / Y} \frac{k \sin \theta \cos \theta^{2}}{k^{2}},
\end{aligned}
$$

where Eqs. (A12) and (A13) have been used and $m^{l_{ \pm}}$is the bare mass of the $l_{ \pm}$pocket whose definition in terms of the Hamiltonian parameters is given by $m^{\Gamma_{ \pm}}=2(a \mp b)^{-1}$ and $m^{X / Y}=m^{e}=2(a+b)^{-1}$. To compute the $k$ integration in Eq. (B9) we evaluate the velocity in Eq. (B12) at the renormalized FS. In the nematic phase $k_{F}(\theta)$ is no longer constant. Using again the expansion up to the first order in $\Sigma_{3}$ of the renormalized energy we can estimate $k_{F}(\theta)$ and replace it in Eq. (B12). We find

$$
\begin{aligned}
V_{R x}^{\Gamma_{ \pm}} & =V_{0_{x}}^{\Gamma_{ \pm}}\left(1 \pm \cos 2 \theta \frac{\operatorname{Re} \Sigma_{3}^{\Gamma}}{2 \epsilon_{0}^{h}} \mp 4 \sin ^{2} \theta \frac{\operatorname{Re} \Sigma_{3}^{\Gamma}}{2 \epsilon_{0}^{h}}\right), \\
V_{R y}^{\Gamma_{ \pm}} & =V_{0 y}^{\Gamma_{ \pm}}\left(1 \pm \cos 2 \theta \frac{\operatorname{Re} \Sigma_{3}^{\Gamma}}{2 \epsilon_{0}^{h}} \pm 4 \cos ^{2} \theta \frac{\operatorname{Re} \Sigma_{3}^{\Gamma}}{2 \epsilon_{0}^{h}}\right), \\
V_{R x}^{X / Y} & =V_{0 x}^{X / Y}\left(1 \pm \cos 2 \theta \frac{\operatorname{Re} \Sigma_{3}^{X / Y}}{2 \epsilon_{0}^{X / Y}} \mp 4 \sin ^{2} \theta \frac{\operatorname{Re} \Sigma_{3}^{X / Y}}{2 \epsilon_{0}^{X / Y}}\right), \\
V_{R y}^{X / Y} & =V_{0 y}^{X / Y}\left(1 \pm \cos 2 \theta \frac{\operatorname{Re} \Sigma_{3}^{X / Y}}{2 \epsilon_{0}^{X / Y}} \pm 4 \cos ^{2} \theta \frac{\operatorname{Re} \Sigma_{3}^{X / Y}}{2 \epsilon_{0}^{X / Y}}\right) .
\end{aligned}
$$

where $\epsilon_{0}^{h}$ and $\epsilon_{0}^{e}$ are the Fermi energy in the tetragonal phase defined in Eq. (A17) and $V_{0_{\alpha}}^{\Gamma_{ \pm}}=-k_{F^{\prime} \alpha}^{\Gamma_{ \pm}} / m^{\Gamma_{ \pm}}, V_{0_{\alpha}}^{X / Y}=$ $k_{0 \% \alpha}^{e} / m^{e}$ is the $\alpha$ component of the bare velocity with $k_{0_{F}}^{\Gamma_{ \pm}}=$ $\sqrt{\epsilon_{0}^{h} /\left(2 m^{\Gamma_{ \pm}}\right)}$and $k_{0}^{e}=\sqrt{\epsilon_{0}^{e} /\left(2 m^{e}\right)}$.

The last term we need to evaluate is the $\left|\nabla \epsilon_{R \mathbf{k}}^{l_{ \pm}}\right|$[see Eq. (B9)]. In the $\Gamma$ pocket turns out to be $\left|\nabla \epsilon_{R \mathbf{k}}^{l_{ \pm}}\right|=k / m^{\Gamma_{+}}$ with similar results for the other pockets. The important point is that the norm of the pocket velocity is independent of the self-energy while the pocket velocity in a given direction $x / y$ depends on the self-energy, Eq. (B12). Replacing all the analytical expressions found for the velocities [Eq. (B12)] and the scattering rate [Eq. (A19)] in Eq. (B9) the pockets dc conductivities read

$$
\begin{aligned}
& \sigma_{x / y}^{\Gamma_{+}}=\sigma^{h}\left(1 \pm \frac{\operatorname{Re} \Sigma_{3}^{\Gamma}}{2 \epsilon_{0}^{h}} \mp \frac{\operatorname{Re} \Sigma_{3}^{\Gamma}}{\epsilon_{0}^{h}} \mp \frac{\left|\operatorname{Im} \Sigma_{3}^{\Gamma}\right|}{2 \Gamma_{0}^{h}}\right), \\
& \sigma_{x / y}^{\Gamma_{-}}=\sigma^{h}\left(1 \mp \frac{\operatorname{Re} \Sigma_{3}^{\Gamma}}{2 \epsilon_{0}^{h}} \pm \frac{\operatorname{Re} \Sigma_{3}^{\Gamma}}{\epsilon_{0}^{h}} \pm \frac{\left|\operatorname{Im} \Sigma_{3}^{\Gamma}\right|}{2 \Gamma_{0}^{h}}\right), \\
& \sigma_{x / y}^{X}=\sigma^{e}\left(1 \pm \frac{\operatorname{Re} \Sigma_{3}^{X}}{2 \epsilon_{0}^{e}} \mp \frac{\operatorname{Re} \Sigma_{3}^{X}}{\epsilon_{0}^{e}} \pm \frac{\left|\operatorname{Im} \Sigma_{3}^{X}\right|}{2 \Gamma_{0}^{e}}\right), \\
& \sigma_{x / y}^{Y}=\sigma^{e}\left(1 \mp \frac{\operatorname{Re} \Sigma_{3}^{Y}}{2 \epsilon_{0}^{e}} \pm \frac{\operatorname{Re} \Sigma_{3}^{Y}}{\epsilon_{0}^{e}} \mp \frac{\left|\operatorname{Im} \Sigma_{3}^{Y}\right|}{2 \Gamma_{0}^{e}}\right),
\end{aligned}
$$

where $\sigma^{h / e}=e^{2} \epsilon_{0}^{h / e} /(2 \pi \hbar) \Gamma_{0}^{h / e}$ is the $x / y$ component of the $\mathrm{dc}$ conductivity in the tetragonal phase for the h/e pocket. The dc conductivity anisotropy for the $\mathrm{h}$ and e pockets can be finally written as

$$
\begin{aligned}
\Delta \sigma_{\mathrm{dc}}^{h+} & =\sigma^{\Gamma}\left(\frac{\Phi^{h}}{\epsilon_{0}^{h}}-\frac{\Delta \Gamma^{h}}{\Gamma_{0}^{h}}\right), \\
\Delta \sigma_{\mathrm{dc}}^{h-} & =\sigma^{\Gamma}\left(-\frac{\Phi^{h}}{\epsilon_{0}^{h}}+\frac{\Delta \Gamma^{h}}{\Gamma_{0}^{h}}\right), \\
\Delta \sigma_{\mathrm{dc}}^{e} & =\sigma^{e}\left(-\frac{\Phi^{e}}{\epsilon_{0}^{e}}+\frac{\Delta \Gamma^{e}}{\Gamma_{0}^{e}}\right),
\end{aligned}
$$

with the nematic order parameters for the h pockets $\left(\Phi^{h}, \Delta \Gamma^{h}\right)$ and the e pockets $\left(\Phi^{e}, \Delta \Gamma^{e}\right)$ defined as

$$
\begin{gathered}
\Phi^{h} \equiv \frac{\operatorname{Re} \Sigma_{x z}^{\Gamma}-\operatorname{Re} \Sigma_{y z}^{\Gamma}}{2}=-\operatorname{Re} \Sigma_{3}^{\Gamma}, \quad \Delta \Gamma^{h} \equiv\left|\operatorname{Im} \Sigma_{3}^{\Gamma}\right|, \\
\Phi^{e} \equiv \frac{\operatorname{Re} \Sigma_{y z}^{X}-\operatorname{Re} \Sigma_{x z}^{Y}}{2}, \quad \Delta \Gamma^{e} \equiv \frac{\left|\operatorname{Im} \Sigma_{y z}^{X}\right|-\left|\operatorname{Im} \Sigma_{x z}^{Y}\right|}{2} .
\end{gathered}
$$

\section{APPENDIX C: MODEL PARAMETERS FOR FESE AND 122 SYSTEMS}

To perform the numerical analysis discussed in the main text we used a set of band parameters which reproduce the experimental dispersions observed in 122 and FeSe systems. Using the band parameters listed in Table I, a spin-orbit interaction of $\lambda=5 \mathrm{meV}$ and $\left|\operatorname{Re} \Sigma_{y z / x z}^{\Gamma}\right|=\operatorname{Re} \Sigma_{y z / x z}^{X / Y}=15 \mathrm{meV}$, we obtain the FS topology shown in Fig. 1 that reproduce qualitatively well the FS of 122 systems in the tetragonal phase. The nematic phase is computed with a symmetric nematic splitting of $\Phi^{h}=\Phi^{e}=4 \mathrm{meV}$. For the FeSe case we used the same set of band parameters listed in Table I, with spin-orbit interaction $\lambda=20 \mathrm{meV}$, and $\left|\operatorname{Re} \Sigma_{y z / x z}^{\Gamma}\right|=$ $70 / 40 \mathrm{meV}$ and $\operatorname{Re} \Sigma_{y z / x z}^{X / Y}=45 / 15 \mathrm{meV}$ that result in a nematic order parameter $\Phi^{h / e}=15 \mathrm{meV}$. This set reproduces the FS and their orbital distribution as experimentally observed by ARPES $[9,16,54]$ in the nematic phase.

We fix the background scattering to $\delta \Gamma=1 \mathrm{meV}$. The scattering rates used in the tetragonal phase for 122 are $\operatorname{Im} \Sigma_{y z / x z}^{\Gamma}=\operatorname{Im} \Sigma_{y z / x z}^{X / Y}=-2 \mathrm{meV}$, while for the FeSe case we used $\operatorname{Im} \Sigma_{y z / x z}^{\Gamma}=-4 \mathrm{meV}$ and $\operatorname{Im} \Sigma_{y z / x z}^{X / Y}=-3 \mathrm{meV}$. In both

TABLE I. Low-energy model parameters used for FeSe and 122 system. All the parameters are in $\mathrm{meV}$, the $k$ vector is measured in units $1 / a \sim 0.375 \AA$, where $a=a_{\mathrm{FeFe}}$ is the lattice constant of the 1-Fe unit cell (so that $\tilde{a}=\sqrt{2} a=3.77 \AA$ is the lattice constant of the $2 \mathrm{Fe}$ unit cell).

\begin{tabular}{lrrrrrr}
\hline \hline & $\Gamma$ & & & & & \\
\hline$\epsilon_{\Gamma}$ & 46 & $\epsilon_{x y}$ & 72 & $\epsilon_{y z}$ & 55 \\
$a_{\Gamma}$ & 263 & $a_{x y}$ & 93 & $a_{y z}$ & 101 \\
$b_{\Gamma}$ & 182 & $b$ & 154 & & & \\
& & $v$ & 144 & & \\
\hline \hline
\end{tabular}


cases their variations of the imaginary part of the self-energies in the nematic phase are assumed to be proportional to the variation of their real parts, i.e., $\Delta \Gamma^{h} \sim c_{h}\left(\Phi^{h} / \operatorname{Re} \Sigma_{0}^{\Gamma}\right)$ and $\Delta \Gamma^{e} \sim c_{e}\left(\Phi^{e} / \operatorname{Re} \Sigma_{0}^{X / Y}\right)$ with $c_{h / e}$ arbitrary coefficients.
[1] Y. Gallais and I. Paul, C. R. Phys. 17, 113 (2016).

[2] R. M. Fernandes, A. V. Chubukov, J. Knolle, I. Eremin, and J. Schmalian, Phys. Rev. B 85, 024534 (2012).

[3] R. M. Fernandes, A. V. Chubukov, and J. Schmalian, Nat. Phys. 10, 97 (2014).

[4] M. C. Rahn, R. A. Ewings, S. J. Sedlmaier, S. J. Clarke, and A. T. Boothroyd, Phys. Rev. B 91, 180501(R) (2015).

[5] Q. Wang, Y. Shen, B. Pan, Y. Hao, M. Ma, F. Zhou, P. Steffens, K. Schmalzl, T. R. Forrest, M. Abdel-Hafiez et al., Nat. Mater. 15, 159 (2016).

[6] M. He, L. Wang, F. Hardy, L. Xu, T. Wolf, P. Adelmann, and C. Meingast, Phys. Rev. B 97, 104107 (2018).

[7] P. Wiecki, M. Nandi, A. E. Böhmer, S. L. Bud'ko, P. C. Canfield, and Y. Furukawa, Phys. Rev. B 96, 180502(R) (2017).

[8] P. Wiecki, K. Rana, A. E. Böhmer, Y. Lee, S. L. Bud'ko, P. C. Canfield, and Y. Furukawa, Phys. Rev. B 98, 020507 (2018).

[9] A. I. Coldea and M. D. Watson, Annu. Rev. Condens. Matter Phys. 9, 125 (2018).

[10] S. Baek, D. Efremov, J. M. Ok, J. S. Kim, J. van den Brink, and B. Büchner, Nat. Mater. 14, 210 (2014).

[11] S. Onari, Y. Yamakawa, and H. Kontani, Phys. Rev. Lett. 116, 227001 (2016).

[12] Y. Su, H. Liao, and T. Li, J. Phys.: Condens. Matter 27, 105702 (2015)

[13] S. Mukherjee, A. Kreisel, P. J. Hirschfeld, and B. M. Andersen, Phys. Rev. Lett. 115, 026402 (2015).

[14] K. Jiang, J. Hu, H. Ding, and Z. Wang, Phys. Rev. B 93, 115138 (2016).

[15] R.-Q. Xing, L. Classen, M. Khodas, and A. V. Chubukov, Phys. Rev. B 95, 085108 (2017).

[16] L. Fanfarillo, J. Mansart, P. Toulemonde, H. Cercellier, P. Le Fèvre, F. Bertran, B. Valenzuela, L. Benfatto, and V. Brouet, Phys. Rev. B 94, 155138 (2016).

[17] A. V. Chubukov, M. Khodas, and R. M. Fernandes, Phys. Rev. X 6, 041045 (2016).

[18] Y. Yamakawa, S. Onari, and H. Kontani, Phys. Rev. X 6, 021032 (2016).

[19] L. Fanfarillo, L. Benfatto, and B. Valenzuela, Phys. Rev. B 97, 121109(R) (2018).

[20] M. A. Tanatar, E. C. Blomberg, A. Kreyssig, M. G. Kim, N. Ni, A. Thaler, S. L. Bud'ko, P. C. Canfield, A. I. Goldman, I. I. Mazin et al., Phys. Rev. B 81, 184508 (2010).

[21] A. Dusza, A. Lucarelli, F. Pfuner, J.-H. Chu, I. Fisher, and L. Degiorgi, Europhys. Lett. 93, 37002 (2011).

[22] I. R. Fisher, L. Degiorgi, and Z. X. Shen, Rep. Prog. Phys. 74, 124506 (2011).

[23] A. Dusza, A. Lucarelli, A. Sanna, S. Massidda, J.-H. Chu, I. R. Fisher, and L. Degiorgi, New J. Phys. 14, 023020 (2012).

[24] C. Mirri, A. Dusza, S. Bastelberger, J.-H. Chu, H.-H. Kuo, I. R. Fisher, and L. Degiorgi, Phys. Rev. B 90, 155125 (2014).

[25] E. C. Blomberg, M. A. Tanatar, R. M. Fernandes, I. I. Mazin, B. Shen, H.-H. Wen, M. D. Johannes, J. Schmalian, and R. Prozorov, Nat. Commun. 4, 1914 (2013).
[26] T.-M. Chuang, M. P. Allan, J. Lee, Y. Xie, N. Ni, S. L. Bud'ko, G. S. Boebinger, P. C. Canfield, and J. C. Davis, Science 327, 181 (2010)

[27] M. Nakajima, S. Ishida, Y. Tomioka, K. Kihou, C. H. Lee, A. Iyo, T. Ito, T. Kakeshita, H. Eisaki, and S. Uchida, Phys. Rev. Lett. 109, 217003 (2012).

[28] S. Ishida, M. Nakajima, T. Liang, K. Kihou, C. H. Lee, A. Iyo, H. Eisaki, T. Kakeshita, Y. Tomioka, T. Ito et al., Phys. Rev. Lett. 110, 207001 (2013).

[29] M. P. Allan, T.-M. Chuang, F. Massee, Y. Xie, N. Ni, S. L. Bud'ko, G. S. Boebinger, Q. Wang, D. S. Dessau, P. C. Canfield et al., Nat. Phys. 9, 220 (2013).

[30] H.-H. Kuo and I. R. Fisher, Phys. Rev. Lett. 112, 227001 (2014).

[31] C. Mirri, A. Dusza, S. Bastelberger, M. Chinotti, L. Degiorgi, J.-H. Chu, H.-H. Kuo, and I. R. Fisher, Phys. Rev. Lett. 115, 107001 (2015).

[32] C. Mirri, A. Dusza, S. Bastelberger, M. Chinotti, J.-H. Chu, H.-H. Kuo, I. R. Fisher, and L. Degiorgi, Phys. Rev. B 93, 085114 (2016).

[33] M. Schütt, J. Schmalian, and R. M. Fernandes, Phys. Rev. B 94, 075111 (2016).

[34] M. Chinotti, A. Pal, L. Degiorgi, A. E. Böhmer, and P. C. Canfield, Phys. Rev. B 96, 121112(R) (2017).

[35] C.-C. Lee, W.-G. Yin, and W. Ku, Phys. Rev. Lett. 103, 267001 (2009).

[36] W. Lv, F. Krüger, and P. Phillips, Phys. Rev. B 82, 045125 (2010).

[37] H. Kontani, T. Saito, and S. Onari, Phys. Rev. B 84, 024528 (2011).

[38] R. M. Fernandes, L. H. VanBebber, S. Bhattacharya, P. Chandra, V. Keppens, D. Mandrus, M. A. McGuire, B. C. Sales, A. S. Sefat, and J. Schmalian, Phys. Rev. Lett. 105, 157003 (2010).

[39] M. Breitkreiz, P. M. R. Brydon, and C. Timm, Phys. Rev. B 90 , 121104(R) (2014).

[40] M. N. Gastiasoro, I. Paul, Y. Wang, P. J. Hirschfeld, and B. M. Andersen, Phys. Rev. Lett. 113, 127001 (2014).

[41] Y. Wang, M. N. Gastiasoro, B. M. Andersen, M. Tomić, H. O. Jeschke, R. Valentí, I. Paul, and P. J. Hirschfeld, Phys. Rev. Lett. 114, 097003 (2015).

[42] S. Liang, G. Alvarez, C. Şen, A. Moreo, and E. Dagotto, Phys. Rev. Lett. 109, 047001 (2012).

[43] B. Valenzuela, E. Bascones, and M. J. Calderón, Phys. Rev. Lett. 105, 207202 (2010).

[44] E. Bascones, B. Valenzuela, and M. J. Calderón, C. R. Phys. 17, 36 (2016).

[45] K. Sugimoto, P. Prelovšek, E. Kaneshita, and T. Tohyama, Phys. Rev. B 90, 125157 (2014).

[46] M. A. Tanatar, A. E. Böhmer, E. I. Timmons, M. Schütt, G. Drachuck, V. Taufour, K. Kothapalli, A. Kreyssig, S. L. Bud'ko, P. C. Canfield et al., Phys. Rev. Lett. 117, 127001 (2016).

[47] S. Onari and H. Kontani, Phys. Rev. B 96, 094527 (2017).

[48] L. Fanfarillo, A. Cortijo, and B. Valenzuela, Phys. Rev. B 91, 214515 (2015). 
[49] J. P. Sun, K. Matsuura, G. Z. Ye, Y. Mizukami, M. Shimozawa, K. Matsubayashi, M. Yamashita, T. Watashige, S. Kasahara, Y. Matsuda et al., Nat. Commun. 7, 12146 (2016).

[50] K. Kothapalli, A. E. Böhmer, W. T. Jayasekara, B. G. Ueland, P. Das, A. Sapkota, V. Taufour, Y. Xiao, E. Alp, S. L. Bud'ko et al., Nat. Commun. 7, 12728 (2016).

[51] L. Benfatto, B. Valenzuela, and L. Fanfarillo, npj Quantum Mater. 3, 56 (2018).

[52] P. O. Sprau, A. Kostin, A. Kreisel, A. E. Böhmer, V. Taufour, P. C. Canfield, S. Mukherjee, P. J. Hirschfeld, B. M. Andersen, and J. C. S. Davis, Science 357, 75 (2017).

[53] Y. S. Kushnirenko, A. V. Fedorov, E. Haubold, S. Thirupathaiah, T. Wolf, S. Aswartham, I. Morozov, T. K. Kim, B. Büchner, and S. V. Borisenko, Phys. Rev. B 97, 180501 (2018).

[54] L. C. Rhodes, M. D. Watson, A. A. Haghighirad, D. V. Evtushinsky, M. Eschrig, and T. K. Kim, Phys. Rev. B 98, 180503 (2018).
[55] D. Liu, C. Li, J. Huang, B. Lei, L. Wang, X. Wu, B. Shen, Q. Gao, Y. Zhang, X. Liu et al., Phys. Rev. X 8, 031033 (2018).

[56] L. de' Medici, Weak and Strong Correlations in Fe Superconductors, Springer Series in Materials Science Vol. 211 (Springer, New York, 2015).

[57] V. Cvetkovic and O. Vafek, Phys. Rev. B 88, 134510 (2013).

[58] M. J. Calderón, L. de' Medici, B. Valenzuela, and E. Bascones, Phys. Rev. B 90, 115128 (2014).

[59] L. Fanfarillo, G. Giovannetti, M. Capone, and E. Bascones, Phys. Rev. B 95, 144511 (2017).

[60] A. F. Kemper, M. M. Korshunov, T. P. Devereaux, J. N. Fry, H.-P. Cheng, and P. J. Hirschfeld, Phys. Rev. B 83, 184516 (2011).

[61] M. M. Korshunov, in Perturbation Theory: Advances in Research and Applications, edited by Z. Pirogov (Nova Science Publishers Inc., New York, 2018), p. 276.

[62] M. Chinotti, A. Pal, L. Degiorgi, A. E. Böhmer, and P. C. Canfield, Phys. Rev. B 98, 094506 (2018). 